\title{
ASGR: An Artificial Spider-Web-Based Geographic Routing in Heterogeneous Vehicular Networks
}

\author{
Chen Chen ${ }^{\circledR}$, Member, IEEE, Lei Liu, Tie Qiu, Senior Member, IEEE, Kun Yang, Senior Member, IEEE, \\ Fengkui Gong, Member, IEEE, and Houbing Song ${ }^{\circledR}$, Senior Member, IEEE
}

\begin{abstract}
Recently, vehicular ad hoc networks (VANETs) have been attracting significant attention for their potential for guaranteeing road safety and improving traffic comfort. Due to high mobility and frequent link disconnections, it becomes quite challenging to establish a reliable route for delivering packets in VANETs. To deal with these challenges, an artificial spider geographic routing in urban VAENTs (ASGR) is proposed in this paper. First, from the point of bionic view, we construct the spider web based on the network topology to initially select the feasible paths to the destination using artificial spiders. Next, the connection-quality model and transmission-latency model are established to generate the routing selection metric to choose the best route from all the feasible paths. At last, a selective forwarding scheme is presented to effectively forward the packets in the selected route, by taking into account the nodal movement and signal propagation characteristics. Finally, we implement our protocol on NS2 with different complexity maps and simulation parameters. Numerical results demonstrate that, compared with the existing schemes, when the packets generate speed, the number of vehicles and number of connections are varying, our proposed ASGR still performs best in terms of packet delivery ratio and average transmission delay with an up to $15 \%$ and $94 \%$ improvement, respectively.
\end{abstract}

Index Terms - Vehicular ad hoc networks, routing protocol, artificial spider-web, connection quality, latency model, selective forwarding.

\section{INTRODUCTION}

$\mathbf{I}$ $\mathrm{N}$ THE recent decades, for the purpose of improving road safety and travel comfort of drivers and passengers, the worldwide governments and societies are active to deploy

Manuscript received June 20, 2017; revised December 27, 2017 and March 13, 2018; accepted April 7, 2018. This work was supported in part by the National Natural Science Foundation of China under Grant 61571338, Grant U1709218, and Grant 61672131, in part by the Key Research and Development Plan of Shaanxi Province under Grant 2017ZDCXL-GY-05-01, in part by the National Key Research and Development Program of China under Grant 2016YFE0123000, in part by the National Science and Technology Major Project of the Ministry of Science and Technology of China under Grant 2015zx03002006-003 and Grant MJ-2014-S-37, and in part by the 111 Project of China under Grant B08038. The Associate Editor for this paper was H. Jula. (Corresponding author: Tie Qiu.)

C. Chen, L. Liu, and F. Gong are with the State Key Laboratory of Integrated Service Networks, Xidian University, Xi'an 710071, China (e-mail: cc2000@mail.xidian.edu.cn; 1liu_136@stu.xidian.edu.cn; fkgong@xidian.edu.cn).

T. Qiu is with the School of Computer Science and Technology, Tianjin University, Tianjin 300350, China (e-mail: qiutie@ieee.org).

$\mathrm{K}$. Yang is with the School of Computer Science and Electronic Engineering, University of Essex, Essex, CO4 3SQ, U.K. (e-mail: kunyang@essex.ac.uk).

H. Song is with the Department of Electrical, Computer, Software, and Systems Engineering, Embry-Riddle Aeronautical University, Daytona Beach, FL 32114 USA (e-mail: Houbing.Song@erau.edu).

Color versions of one or more of the figures in this paper are available online at http://ieeexplore.ieee.org.

Digital Object Identifier 10.1109/TITS.2018.2828025 and realize the Intelligent Transportation Systems (ITS). Among those popular technologies, the Vehicular Ad Hoc Network (VANET) represents a substantially significant research direction in recent years, which mainly stems from its high potential to the ITS [1]. VANETs define the moving vehicles or road facilities as the nodes, and enable information to be exchanged between vehicles (V2V) or between vehicles and road facilities (V2I). However, due to frequent changes of network topology, constraints of road layouts and blocking of wireless signals by obstacles, designing one routing protocol in urban vehicular environments is more complex than in Mobile Ad Hoc Networks (MANETs). In fact, the aforementioned issues may cause frequent network disconnections, which is the most fatal problem for a routing protocol. As a result, traditional topology-based routing protocols designed for MANETs may not be suitable in vehicular communications. An alternative approach is offered by the positionbased routing protocols with the help of information obtained from geographic information services and navigation systems [2]. These position-based protocols usually take the road constrains, wireless signal obstacles, traffic lights and traffic density into consideration at the same time [3].

On the other hand, the multi-hop transmission is quite important for a lot of applications in VANETs. For example, a moving vehicle can query a data center several miles away for traffic status by multi-hop V2V communications; the drivers or passengers who intend to shop at a faraway mall may query the roadside buffer of this store by multi-hop V2V communications for some interested goods; a driver can engage a wise route to reach the available parking lot according to the information shared by neighboring vehicles. Although the promising $\mathrm{V} 2 \mathrm{~V}$ communication pattern has attracted many research efforts in the last few years, most previous works intend to assume an ideal scenario in which the network is well connected, with no or only few network partitions. However, recent researches have shown that the network fragmentation problem is much severe in VANETs, especially for sparse traffic environments. Even during rush hours, due to the low penetration ratio of equipped vehicles on the road, the network has a lower connectivity. In addition, the high nodal mobility and dynamic topology change will further ruin the network connectivity. In this situation, one complete connected endto-end route is difficult to be built. To solve this issue, when link partitions happen, the carry-and-forward scheme is usually employed to forward data packets, by which the data can be buffered first and forwarded by the relay vehicles opportunistically, in case that an available next hop is acquirable. 
According to above analysis, it can be concluded that the feasibility of VANET applications relying on V2V pattern is mainly subjected to the network connectivity along a routing path, with the premise that they can be finished before a predefined deadline. In other words, the main objective of a $\mathrm{V} 2 \mathrm{~V}$ routing is to maximize the data delivery ratio while minimizing the end-to-end transmission delay at the same time. As a result, two challenging issues arise: 1) how to select the best route to the destination with connectivity and delay considered; 2) how to forward data hop by hop along the selected route. It is worth noting that the applications considered in our work are commercial or entertainment related, while the safety or emergency related services are not addressed by our protocol in view of their high reliability and time constraint requirements. Actually, for those safety-related applications, the most feasible solution is direct broadcasting, including CAM (Cooperative Awareness Messaging), DENM (Decentralized Environmental Notification Messaging) and BSM (Basic Safety Messaging) etc [4].

On the other hand, based on the similarity of the communication model between vehicles and the natural individuals of a species, many bio-inspired algorithms have been put forward to solve the emerged issues in vehicular environments, especially for routing problems [5], [6]. Inspired by the ideas behind these works, a bio-inspired, intersection-based, multihop geographic routing is also proposed in our work, with the aforementioned requirements of applications and limitations of VANETs considered. According to the fact that a spider can quickly spot and approach the prey trapped in the web, our routing protocol is designed to imitate spiders to find an available path to the prey regardless of the prey's position on the spider-web. Starting with the above motivation and from the point of bionic view, we further distill an idea through the study to the behavior of spiders to construct an artificial spider-web, by which all available routing paths composed of intersections can be determined. After the initial selection of feasible routes by the spider-web, we further add two constrains to the available routes in terms of connection quality and transmission delay, by which an optimal path could be extracted with the expected performance. Finally, a selective forwarding scheme is presented to deliver data along the selected path.

In summary, the main contributions of our work can be generalized as follows:

- The source can determine all the feasible paths to the destination by using the virtual spiders, without the necessity to send traditional RREQ and reply RREP. In this way, the communication overheads can be reduced to a great extent.

- We propose a routing selection metric named as connection quality which combines the connection degree with the connection probability among vehicles on a road segment. In addition, a transmission latency model is proposed as a function of the vehicular densities, speeds, the length of the road segment and the corresponding number of lanes. With the proposed connection quality and transmission latency model, an optimized route from the source to the destination is determined, in order to guarantee that the packets are transmitted along a path that is firmly connected with as short delay as possible.

- Upon receiving a packet, the relay node will forward the packet with our proposed selective forwarding scheme, taking into account the features of data forwarding on road segments and at intersections.

The remainder of the paper is organized as follows. Some related works are discussed in Section II. Assumptions and system model are given in Section III. In Section IV, we depict the framework of our proposed protocol. How to determine the feasible paths is presented in Section V. The approach of selecting the optimized path is proposed in Section VI. The packet forwarding procedure is introduced in VII. In Section VIII, the protocol optimization scheme is proposed. The performance evaluation is given via simulations in Section IX, followed by the conclusion in Section X.

\section{RELATED WORK}

In this section, we will review some typical routing schemes in VANETs. In addition, some bio-inspired routing protocols are also generalized for further comparisons with our work.

A lot of routing protocols make full use of the geographic information to forwarding data packets. GPSR [7] uses greedy forwarding to forward packets initially. When a packet reaches a local maximum, it will not change back until reaching to a node closer to the destination. Accordingly, greedy forwarding is unsuitable for high dynamic scenarios and may not be able to maintain the next-hop neighbors' information. CAR [8] addresses the above problems by selecting an optimized route with the least probability of network disconnection and avoiding the carry-and-forward delay. At the same time, CAR mentions the impact of traffic lights on the connectivity, but it does not apply this result to the routing protocol's design. What's more, the inaccuracy of road density measurement will affect the path selection. In view of above shortcomings, ACAR [9] proposes an on-the-fly density collection process to improve the inaccuracy of CAR.

Intersection-based routing protocols are more effective and stable for urban VANETs. IGRP [10] chooses the path that maximizes the connectivity probability while satisfying the QoS (Quality of Service) constraints. Between any two intersections on the selected path, the geographical forwarding is used to transfer packets, thus reducing the path's sensitivity to individual node movement. However, to reach this goal, IGRP uses the genetic algorithm to choose the optimized route. Therefore, the computation complexity and convergence speed of the genetic algorithm should also be taken into account in the process of routing design which may fail to provide some delay-sensitive services. JBR [11] makes use of the selective greedy forwarding up to the coordinator node that is located at a junction and is closer to the destination. If there is any available coordinator along the routing path, it will be selected in priority and the one closest to the destination is chosen as the next hop. However, since the broadcasting to multiple coordinators for generating multiple paths is prohibited to reduce the experienced delay, the probability of local optimum and packet drop increases in their work. To overcome this 
problem, a junction-based multi-path source routing algorithm is proposed in [12]. Its performance evaluations show that multi-path routing is much beneficial for VANETs, in case the distances between two nodes are medium or long, or the traffic loads are medium to high. Nevertheless, how to handle the local optimum is not yet considered in this paper.

Many routing protocols develop different routing metrics to determine the optimal routing path for data forwarding. A Connectivity-aware minimum-delay Geographic Routing (CMGR) protocol is researched in [13]. When the network is sparse, it takes the connectivity of road sections in consideration, in order to maximize the probability of packets reception. If the network is dense, it reduces the delay by selecting non-congested routes that have a sufficient level of connectivity over time. However, it has no efficient repair strategy to solve significant packet losses. SRCP [14] builds a stable backbone on each road segment which is connected at intersections with the assistance of bridge nodes. According to the information collected via bridge nodes, the end-toend delay for each routing path between nodes can be given before sending data packets. Then, the one with lowest delay is selected as the optimized route. Given the considerable size of modern cities, it is extremely difficult to have a global knowledge of the network topology. Thus, as one extension to SRCP, one articulation junction selection is further proposed in [15], where the city is divided into different zones and an articulation junction in each zone is elected. The articulation junction is defined as the intersection which is connected to most of the intersections in its respective zone. Each elected articulation junction can have a global view of the zone's topology, which will help in making the optimal route selection.

By exploring the broadcast nature of wireless channel and the diversity of packet reception, opportunistic routing can improve the multi-hop communication reliability. LLA [16] provides stable communication paths to deal with the stability of any-path communications. Its key novelty is to the proposal of one special metric of link cost that combines the packet delivery ratio and link stability information. However, it cannot perform well in the high density environment. SPRE [17] introduces a novel concept of link correlation to reflect the impact of relative link positions in a network topology on the network performance. With the concept, a routing metric named as expected transmission cost is designed as the selection guidance of the best route. Based on the metric, a streetcentric opportunistic routing protocol is proposed.

Due to the similarity of the communication model between vehicles and the natural behaviors of a species' individuals, many bio-inspired algorithms are put forward for routing design. MAR-DYMO [18] adopts the mobility-aware Ant Colony Optimization algorithm to enhance the Dynamic MANT On-demand routing protocol, where the information about the location and velocity of one vehicle is utilized to modify HELLO messages to allow other vehicles to make the prediction of their mobility. However, the protocol doesn't take the unpredicted changes which may occur to the established routes into consideration. HyBR [5] combines the feature of topology routing with those of geographic routing, applying two procedures to deal with the varying density. However, each node needs to save the position information of all VANET nodes in a table called a position table which is updated whenever the network topology changes, and possess its routing table that contains the various routes toward the desired destination, which increases its overhead load.

\section{ASSumptions AND System Model}

In this section, our system model will be first given with some reasonable assumptions.

\section{A. Assumptions}

In our work, nodes in VANETs are capable of obtaining their positions and speeds with the help of GPS. Moreover, GPS errors can be minimized by means of various standard procedures, e.g., augmentation, precise monitoring, timekeeping, and carrier phase tracking etc. Meanwhile, the topology of all road sections in the city is gotten via the onboard electronic map which can provide street-level map details and time-dependent traffic statistics, including density and average speed of vehicles on different road segments, such as MapMechanics. Further, every source node is able to get the transient geographical position of the destination by a location service e.g., RLSMP [19]. Note that the above assumptions are reasonable and feasible with present technologies and have been adopted by a lot of work [15], [20].

\section{B. System Model}

1) Signal Fading Model: In our work, the Nakagami-m distribution is used to describe the fading of radio wave propagation. The successful transmission probability of data packets between vehicle $i$ and $j$ against channel fading can be given by [17]:

$$
p_{i j}^{f}=1-F_{d}\left(r_{T} ; m, \psi\right),
$$

where $F_{d}\left(r_{T} ; m, \psi\right)$ indicates the cumulative distribution function of receiving signal power, $r_{T}$ represents the reception threshold of a signal, $\psi$ is an average power strength, and $m$ stands for the fading parameter which is a function of the inter-vehicle distance between vehicle $i$ and $j$.

2) Vehicular Traffic Model: Suppose a two-way road as shown in Fig. 1, where vehicles are moving in two opposite directions on the road segment $i$ of length $L_{i}$. Assume that the vehicles on both lanes are uniformly distributed with node spatial density $\rho_{1}$ for lane 1 and $\rho_{2}$ for lane 2 [10], respectively. Then, the probability that $k$ vehicles are falling into a segment of length $l$ on each lane $\vartheta(\vartheta=1,2)$ follows a Poisson distribution as: $f_{\vartheta}(k, l)=\frac{\left(\rho_{\vartheta} l\right)^{k}}{k !} e^{-\rho_{\vartheta} l}$. The probability that the inter-vehicle distance between two adjacent vehicles is smaller than $x$ on lane $\vartheta$ follows an independent and identically distributed (i.i.d) exponential distribution as:

$$
\begin{aligned}
F_{\vartheta}(x) & =P\{X<x\}=1-f_{\vartheta}(0, x) \\
& =1-e^{-\rho_{\vartheta} x}, \quad x>0,
\end{aligned}
$$

and the probability density function (PDF) can be derived as:

$$
f_{\vartheta}(x)=F_{\vartheta}{ }^{\prime}(x)=\rho_{\vartheta} e^{-\rho_{\vartheta} x}, \quad x>0 .
$$




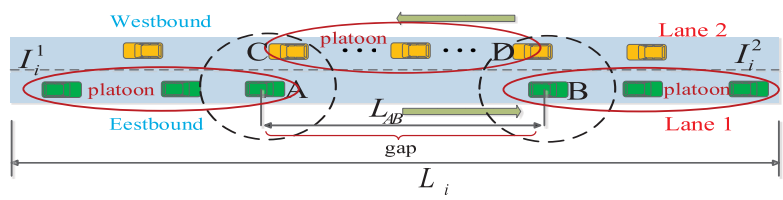

Fig. 1. An example of the road segment.

A platoon can be formed by a series of vehicles in the same lane if each vehicle is within the adjacent vehicle's radio range denoted by $R$. In this platoon, the PDF of the inter-vehicle distance can be expressed as: $f_{\vartheta}(l \mid l<R)=F_{\vartheta}{ }^{\prime}(l \mid l<R)=$ $\frac{\rho_{\vartheta} e^{-\rho_{\vartheta} l}}{1-e^{-\rho_{\vartheta} R}}$, and its expectation is: $E\left[d_{\text {int } r a}^{\vartheta}\right]=\frac{1}{\rho_{\vartheta}}-\frac{R e^{-\rho_{\vartheta} R}}{1-e^{-\rho_{\vartheta} R}}$. From [21], it is known that the expected platoon length is written as: $E\left[C_{\text {platoon }}^{\vartheta}\right]=\left(e^{\rho_{\vartheta} R}-1\right)\left(\frac{1}{\rho_{\vartheta}}-\frac{R e^{-\rho_{\vartheta} R}}{1-e^{-\rho_{\vartheta} R}}\right)$. For two adjacent platoons, their inter-vehicle distance, namely, the gap between them, is larger than $R$. The link between them is defined as broken, e.g., the link between A and B on lane 1 in Fig. 1. In this case, the PDF of the inter-vehicle distance for a broken link can be formulated as: $f_{\vartheta}(l \mid l>R)=$ $F_{\vartheta}(l \mid l>R)=\rho_{\vartheta} e^{-\rho_{\vartheta}(l-R)}$, and its expectation is:

$$
E\left[d_{\text {inter }}^{\vartheta}\right]=R+\frac{1}{\rho_{\vartheta}} .
$$

3) Link Connection Model: Define the link connection time $\operatorname{link}_{i j}$ between vehicle $i$ and $j$ as the duration at which the distance between them is still smaller than the radio range. The elapsed time from $t$ before a disconnection occurs between $i$ and $j$ can be further estimated as [22]:

$$
\operatorname{lin} k_{i j}=\frac{H-\theta \sqrt{\left(y_{i}-y_{j}\right)^{2}+\left(x_{i}-x_{j}\right)^{2}}}{\left|v_{i}-\vartheta v_{j}\right|},
$$

where $v_{i}$ and $v_{j}$ indicate the speeds of vehicle $i$ and $j$, respectively, $\left(x_{i}, y_{i}\right)$ and $\left(x_{j}, y_{j}\right)$ represent their the coordinate positions, $H$ is the inter-vehicle distance. When vehicle $j$ overtakes vehicle $i, \theta=-1$ and $\vartheta=1$; When vehicle $j$ moves forward in front of vehicle $i, \theta=1$ and $\vartheta=1$; When vehicle $i$ and $j$ are moving toward each other, $\theta=-1$ and $\vartheta=-1$; When vehicle $i$ and $j$ are moving away from each other, $\theta=1$ and $\vartheta=-1$.

4) Channel Contention Model: Our proposed protocol employs the IEEE 802.11p MAC protocol based on the DCF . Let $E\left[X_{p}\right]$ represent the mean value of time slots needed for successfully forwarding a packet, and $E\left[s_{p}\right]$ the mean length of each time slot. Then, the transmission delay required denoted by $E\left[D_{p}\right]$ for a successful packet transmission between two nodes, provided that the back-off stage does not exceed the maximum value $m$, can be given by [23]:

$$
E\left[D_{p}\right]=E\left[X_{p}\right] E\left[s_{p}\right]
$$

where

$$
\left\{\begin{array}{l}
E\left[s_{p}\right]=\left(1-P_{t r}\right) \sigma+P_{t r} P_{s} T_{s}+P_{t r}\left(1-P_{s}\right) T_{c} \\
E\left[X_{p}\right]=\frac{\sum_{i=0}^{m}\left(p_{b}{ }^{i}-p_{b}{ }^{m+1}\right) \frac{W_{i}}{2}}{1-p_{b}{ }^{r}} .
\end{array}\right.
$$

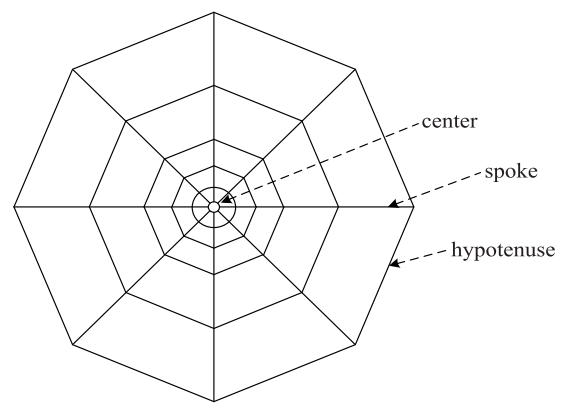

Fig. 2. Spider-web structure.

$P_{t r}$ is the probability that at least one node transmits the packet in one slot time denoted by $\sigma: P_{t r}=1-(1-\tau)^{n}$ and $P_{S}$ is the probability that an occurring packet transmission is successfully given: $P_{S}=\frac{n \tau(1-\tau)^{n-1}}{1-(1-\tau)^{n}} \cdot p_{b}$ indicates the packet collision probability: $p=1-(1-\tau)^{n-1}$, where $\tau$ is the transmission attempt probability. $W_{i}$ represents the contention window size in the $i^{\text {th }}$ back-off stage. $T_{S}$ and $T_{c}$ are the average times that the medium is sensed busy due to a successful transmission or a collision, respectively.

\section{The Framework OF OUR Proposed Protocol}

The spider-web can be considered centrally symmetric with its structure as shown in Fig. 2. It mainly consists of the spokes and hypotenuses. The spokes start from the center of the web, and the hypotenuses refer to the spiral sides around the center.

In the nature, it is found that a spider can find an optimal path by the spider-web to discover the prey. Based on the behavior research of the spiders, we propose one novel geographic routing protocol ASGR in urban VANETs from the point of bionic view. The idea behind ASGR is given as follows:

1) Determine the feasible paths. With the help of GPS, digital map and location service, the artificial spiders are generated to get the information of all the available paths to the destination. By these information collected, the path tree can be built. Though the path tree, the depth-first traversal algorithm is employed to all the available routes to the destination.

2) Select the optimized path. Among all the available paths, the source node selects the optimized path based on the connection-quality model and transmission-latency model.

3) Packet forwarding strategy. Once determining the optimized path, one selective forwarding strategy is developed to forward data packets.

4) Protocol optimization scheme. The road segment information update scheme is presented to guarantee the accuracy of the obtained traffic information. The position tracking scheme is proposed to deal with the mobility of nodes.

\section{Determine the Feasible Paths}

In this section, the source node can construct the artificial spider-web by collecting the information of all intersections through the artificial spiders, with the help of the navigation 


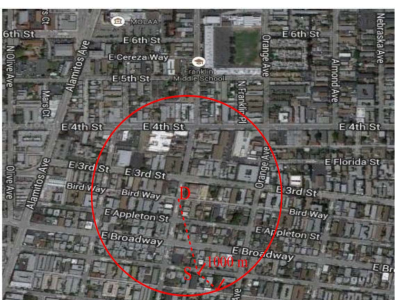

Fig. 3. One selected scenario.

system (e.g. the digital map and GPS) and the location service. Based on the spider-web, the path tree can be built. Through the path tree, all the available paths between two nodes can be determined by employing the depth-first traversal algorithm. It should be noticed that the artificial spiders used are actually virtual and not real probe messages. Thus, during the process, route request and route reply are not used, greatly reducing the communication overheads.

With the objective of reducing the computational complexity, we try to find the optimal routing path by placing a boundary to restrict the searching zone, including the source and destination node. The boundary used in this paper is one circle, with its center in the destination node. A large boundary covering more road segments can generally generate more close-tooptimal routing paths but more computation overhead. Thus, there is one tradeoff. Here, referring to [24], the circle's radius is the distance between the source and the destination node plus $1000 \mathrm{~m}$, by considering the computational complexity, application requirements and city environments, e.g., the city topology, the number of junctions, the average length of road segments, as shown in Fig. 3, where $\mathrm{S}$ denotes the source node, D denotes the destination.The $1000 \mathrm{~m}$ configuration corresponds to the large size city, as Washington DC and Rio evaluated in our simulations. In case of small size cites, the $1000 \mathrm{~m}$ configuration can be reduced to a reasonable range in order to be adaptable to the area for searching. Actually, there are a lot of approaches to place the boundary as long as the destination is enclosed, e.g, [24] and our previous work [3]. Therefore, how to place the boundary is not the major concern of the paper, and it does not affect the correctness of our proposed algorithm.

Next, we introduce the procedure of determining the feasible paths in detail.

\section{A. Construct the Spider-Web}

Step 1 (Determine the Source Intersection and the Destination Intersection According to Their Respective Positions): The source intersection: In order to guarantee the direction of data transmission and reduce the delay as soon as possible, the intersection of the road segment where the source node is located which is closer to the destination node is chosen as the source intersection.

The destination intersection: Two intersections of the road segment where the destination is located are defined as the destination intersections. So, there are usually two destination intersections. However, if the destination node is just located at the intersection by chance, there is only one destination intersection.

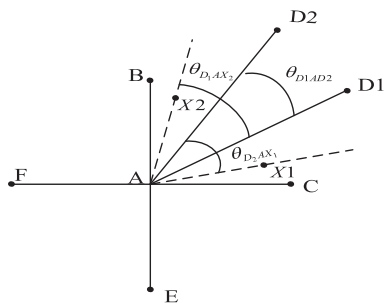

Fig. 4. Determine the intersections.

Step 2 (Send the First-Generation Forward-Spiders Toward the First-Layer Intersections Determined by the Source Intersection): At the beginning, the first-generation forward-moving spiders are generated to move toward the first-layer intersections. During the progress, it is important to determine the first-layer intersections. The detailed method is introduced as follows.

$\mathrm{A}$ is the source intersection, $\mathrm{B}, \mathrm{C}, \mathrm{E}, \mathrm{F}$ represent its adjacent intersections, respectively, and D1 and D2 are the two destination intersections, as shown in Fig. 4. Without loss of generality, assume that X1 and X2 are the two first-layer intersections. Then, the road segment between intersection $\mathrm{A}$ and $\mathrm{X} 1$ or $\mathrm{X} 2$ is defined as the boundary spoke. X1 and X2 are determined as the two first-layer intersections if and only if the conditions given here hold:

1) The destination intersections should be within the searching area formed by the two boundary spokes $A X 1$ and $A X 2$. In other words, the following inequalities need to hold true:

$$
\theta_{D_{1} A D_{2}} \leq \theta_{X_{1} A D_{2}} \text { and } \theta_{D_{1} A D_{2}} \leq \theta_{D_{1} A X_{2}}
$$

Note that when $\theta_{D_{2} A X 1}=\theta_{D_{2} A D 1}\left(\right.$ or $\theta_{D_{1} A X 2}=$ $\theta_{D_{1} A D_{2}}$ ), the corresponding intersection $\mathrm{X} 1$ (or $\mathrm{X} 2$ ) is the only one first-layer intersection.

2) If there may exist many pairs of intersections which meet the condition above, the searching area formed by $A X 1$ and $A X 2$ should be the minimum range, namely, $\left|\theta_{A X 1}-\theta_{A X 2}\right|$ should be the minimum angle.

By this way, $\mathrm{B}$ and $\mathrm{C}$ are determined as the two first-layer intersection.

Step 3: Send out the $(i+1)^{\text {th }}$ generation forward-moving spiders from the $i^{\text {th }}$ layer intersections in their searching area.

Definition 1: The spiders generated by the source intersection are defined as the first-generation forward-spiders.

Definition 2: The intersections which the $i^{\text {th }}(i \geq 1)$ generation forward-moving spiders are intended to reach are defined as the $i^{\text {th }}$ layer intersections.

Definition 3: The spiders generated by the $i^{\text {th }}(i \geq 1)$ layer intersections are defined as the $(i+1)^{t h}$ generation forwardmoving spiders.

When the $i^{\text {th }}(i \geq 1)$ generation forward-moving spiders arrive the corresponding $i^{\text {th }}$ layer intersections, the same procedure as illustrated in Step 2 will be executed by each $i^{\text {th }}$ layer intersection to determine the $(i+1)^{t h}$ layer intersections and then the $(i+1)^{t h}$ generation forward-moving spiders are send out toward the $(i+1)^{t h}$ layer intersections. During the process, the following rules need to be implemented: 
1) If the $(i+1)^{t h}$ layer intersection determined is out of the restricted zone, the $i^{\text {th }}$ generation spider located in the $i^{\text {th }}$ intersection does not send out the next generation spider to the corresponding $(i+1)^{t h}$ layer intersection;

2) If the $(i+1)^{t h}$ layer intersection determined is same with the previous layer intersection, then the current $i^{\text {th }}$ generation spider located in the $i^{\text {th }}$ layer intersection does not send out the next generation spider to the corresponding $(i+1)^{t h}$ layer intersection;

3 ) If the $i^{t h}$ generation spider sends out all the next generation spiders, it will die.

Step 4 (Repeat Step 3 Until the Forward-Moving Spiders Search Out All the Destination Intersections): When the forward-moving spider reaches one destination, it will die, and no next generation spider is generated.

Step 5: After the digital map is searched, the backwardmovin spiders return all the search paths. Then the source intersection constructs the spider-web using the collected information.

\section{B. Generate the Path Tree}

After obtaining the spider-web structure, we can build one set consisting of all the intersections in the structure. Then, a path tree can be constructed using the rules as follows:

1) After an intersection is added to the path tree, delete this intersection from the intersections set it belongs to.

2) The children of the intersection in $(i+1)^{t h}$ layer can only be its neighboring intersections in the boundary spokes of the anterior $i^{\text {th }}$ layer.

3) If the source intersection is selected as the children, the process for constructing the path tree terminates.

4) When the intersections set is NULL, the construction of all the path trees complete.

With the above rules, a path tree can be generated by the following steps:

1) Initialize the path tree to NULL. Add one destination intersection to the path tree as the root.

2) Add the children of the destination intersection from the intersection set to the different leaf with the same level of the path tree.

3) Add respectively the children to the new leaf in 2) at the same time to the path tree in the same way in 2). Repeat the process until the source is selected as the children, then the path tree is constructed completely.

\section{Find All the Feasible Paths}

According to the algorithm above, the path trees rooted with each destination intersection will be generated. Based on each generated path tree, the depth-first traversal algorithm will be performed to find all the available paths with the root as the starting point and the leaf intersection as the ending point, the reverse paths of which are the feasible paths from the source to the destination node.

\section{A Case Study}

Next, take one simple scenario as illustrated in Fig. 5 for example to further elaborate the whole progress stated

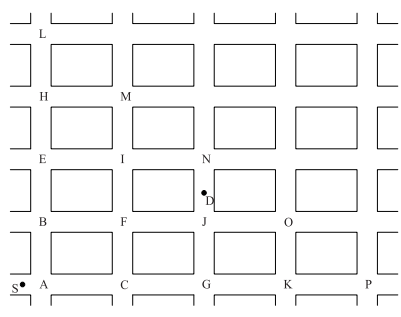

Fig. 5. Example of one scenario.

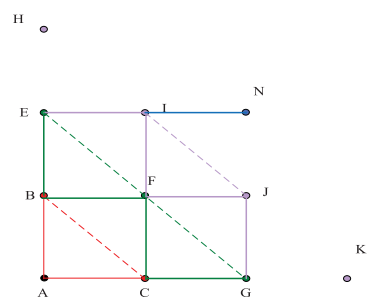

Fig. 6. Spider-web structure.

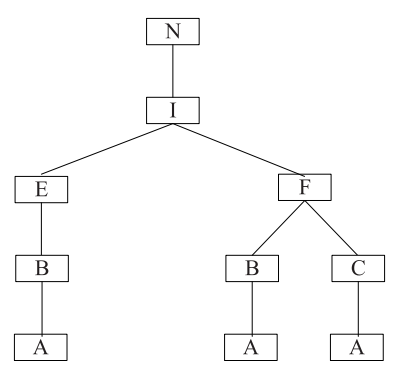

(a)

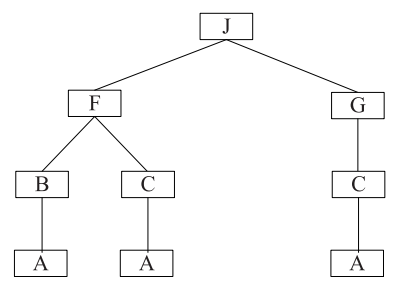

(b)
Fig. 7. Structure of the tree rooted at the destination intersection. (a) At destination intersection N. (b) At destination intersection J.

above, where $\mathrm{S}$ is source node and $\mathrm{D}$ is the destination node. With the searched paths and the contiguity relations between intersections, we can plot the spider-web structure in Fig. 6 related to the topology in Fig. 5, where the points with the same color denote the intersections of the same layer and are connected with the dotted line that represents the boundary spoke of the searching area. The red lines, green lines, purple lines, blue lines are the searching paths of the first-layer, the second-layer, the third-layer and the fourth-layer by the forward spider, respectively.

According the spider-web structure, the path trees rooted at the destination intersections $\mathrm{N}$ and $\mathrm{J}$ can be built as illustrated in Fig. 7(a) and Fig. 7(b), respectively. By using the depthfirst traversal algorithm, for Fig. 7(a), there are 3 paths to the destination intersection N, i.e., ABEIN, ABFIN and ACFIN. Similarly, in Fig. 7(b), there are 3 paths to the destination intersection, i.e., ABFJ, ACFJ and ACGJ.

\section{Select the Optimized Path}

With the obtained feasible paths, an optimized path from them should be selected to forward data packets. In order to achieve this goal, in this section we first propose the connection-quality model and transmission-latency model, and then based on these two models, an optimized path selection algorithm is designed. 


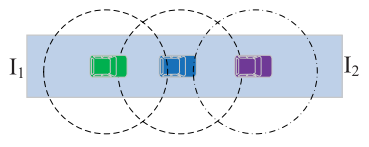

(a)

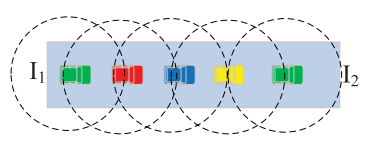

(b)
Fig. 8. Example of connection probability of vehicles. (a) Case a. (b) Case b.

\section{A. Connection Quality}

Our work is focused on the light-traffic environment. Due to the dynamic changing topology and various densities which depend on the time of day or day of the week, a vehicular network is expected to suffer from repetitive network partitioning and link disruptions thus making the messages exchanging difficult among vehicles. As a result, the connectivity plays a key role in the improvement of packet delivery ratio and throughput [25]. However, traditional methods often utilize the connection probability of all vehicles on one lane, say the probability that each vehicle is within the radio range of the adjacent one to indicate the network connectivity, without taking the connection distance of vehicles into consideration. As shown in Fig.8, for case "a" and case "b", if each vehicle is within the transmission range of the adjacent vehicle, the link consisting of all the vehicles in each lane with the same length of road segment is connected. By traditional methods, the connection probability of each case is 1 and both of them have the same connectivity. But it is found that by comparison with case $a$, the connection distance of the link in case b is larger. If there exists one packet which is sent from junction $I_{1}$ to $I_{2}$, the delay needed for case $\mathrm{b}$ is smaller than case a. So, in the section, we propose one new connectivity index called connection quality which makes use of the combination of the connection probability and the connection degree as follows:

$$
P_{c q}=P_{c p} \cdot P_{c d}(\alpha),
$$

where $P_{c q}$ denotes the connection quality of the road segment, $P_{c p}$ indicates the connection probability of vehicles on the road segment, that is the probability that all the vehicles are connected, $S_{c d}$ is the connection degree of the road segment which is defined as the probability that the ratio of the connection distance of the road segment $S_{c d}$ to the road segment's length $L_{i}$ is bigger that one value $\alpha$ when the road segment is full connected, i.e.,

$$
P_{c d}(\alpha)=\operatorname{Pr}\left(\frac{S_{c d}}{L_{i}}>\alpha\right) .
$$

1) Connection Degree: Let $N_{\vartheta}$ denote the number of vehicles on one lane and $x_{i}\left(i=1,2, \ldots, N_{\vartheta}-1\right)$ represent the inter-vehicle distance between two consecutive vehicles, then the sum of all the inter-vehicle distances is: $S_{N_{\vartheta}}=\sum_{i=1}^{N_{\vartheta}-1} x_{i}$.

Based on Equation (3), we can get the characteristic function of $x_{i}$ as:

$$
\begin{aligned}
\varphi_{x_{i}}(t) & =\int_{0}^{+\infty} f_{\vartheta}(u) e^{j t u} d u \\
& =\int_{0}^{+\infty} \rho_{\vartheta} e^{-\rho_{\vartheta} u} e^{j t u} d u \\
& =\frac{\rho_{\vartheta}}{\rho_{\vartheta}-j t} .
\end{aligned}
$$

Further, the characteristic function of $S_{N_{\vartheta}}$ can be attained:

$$
\varphi_{S_{N_{\vartheta}}}(t)=\left[\varphi_{x_{i}}(t)\right]^{N_{\vartheta}-1}=\left(\frac{\rho_{\vartheta}}{\rho_{\vartheta}-j t}\right)^{N_{\vartheta}-1} .
$$

Then, the PDF of $S_{N_{\vartheta}}$ is

$$
\begin{aligned}
f_{S_{N_{\vartheta}}}(x) & =\frac{1}{2 \pi} \int_{-\infty}^{+\infty} e^{-j t x} \varphi_{S_{N_{\vartheta}}}(t) d t, \quad x>0 \\
& =\rho_{\vartheta} e^{-\rho_{\vartheta} x} \frac{\left(\rho_{\vartheta} x\right)^{N_{\vartheta}-2}}{\left(N_{\vartheta}-2\right) !} .
\end{aligned}
$$

Correspondingly, the cumulative distribution function (CDF) is formulated by:

$$
\begin{aligned}
F_{S_{N_{\vartheta}}}(x) & =\int_{0}^{x} f_{S_{N_{\vartheta}}}(x) d x \\
& =1-\sum_{k=0}^{N_{\vartheta}-2} \frac{\left(\rho_{\vartheta} x\right)^{k}}{k !} e^{-\rho_{\vartheta} x}, \quad x>0 .
\end{aligned}
$$

As a result, according to Equation (9), the connection degree of the road segment when the road segment is full connected is:

$$
P_{c d}(\alpha)=\operatorname{Pr}\left(\frac{S_{c d}}{L_{i}}>\alpha\right)=1-F_{S_{N_{\vartheta}}}\left(L_{i} \alpha\right) .
$$

2) Connection Probability: In this subsection, based on the relationship between the transmission range and the length of the road segment, we give the following two cases to analyze the connection probability.

Case 1 (The Road Segment's Length Is Small Than R): In this case, since the distance between any two consecutive vehicles is smaller than the radio range, the link consisting of all the vehicles on the road segment is connected. Namely, the link's connection probability is: $p_{c p}=1$.

Case 2 (The Road Segment's Length Is Greater Than R): Considering the dynamic characteristics of a VANET, it is more likely that multiple broken links can exist on a lane. A vehicle may be able to take advantage of the vehicles moving in the opposite direction to complete the information forwarding. From [21], the expectation of the number of broken links on one lane can be expressed as:

$$
N_{\text {gap }}^{\vartheta}=\frac{L_{i}}{E\left[C_{\text {platoon }}^{\vartheta}\right]+E\left[d_{\text {inter }}^{\vartheta}\right]} .
$$

Without loss of generality, we first analyze the performance of the broken link between A and B on lane 1, and then give the model of the connect probability of the whole road segment via multi-hop transmissions.

Since the radio range of vehicles is generally far greater than the width of the road segment, for convenience, we can map A, B into virtual A1, B1 on the corresponding positions of the adjacent other lane 2 respectively, as illustrated in Fig.9. Suppose $N_{A B}$ as the number of vehicles between $\mathrm{A} 1$ and $\mathrm{B} 1$, with $z_{w 1}$ as the first vehicle and $z_{w n}$ as the last one. If the link between $\mathrm{A} 1$ and $z_{w 1}$ is connected, meanwhile the distance $S_{N_{A B}}$ between them meets the following condition: $L_{A B}-R<S_{N_{A B}}<L_{A B}$, then $\mathrm{A}$ and $\mathrm{B}$ can connect with each other through multi-hop forwarding with the help of vehicles located in the coverage gap $L_{A B}$ on lane 2 . 


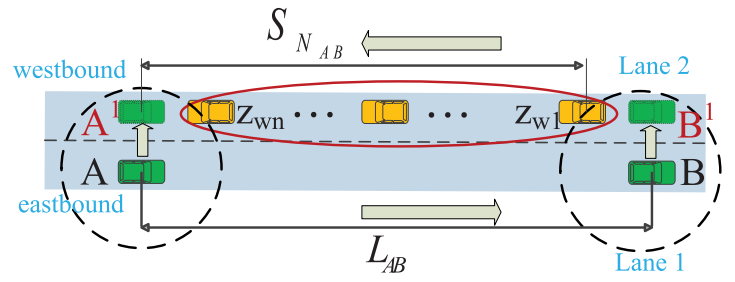

Fig. 9. Example of modeling the connection probability.

To simplify the calculation, according to Equation (4), $L_{A B}$ is set as an average value: $L_{A B}=R+\frac{1}{\rho_{1}}$.

According to Equation (2), the probability denoted by $P_{c}$ that any pair of vehicles between $\mathrm{A} 1$ and $z_{w 1}$ is connected is: $P_{c}=F_{2}(R)=1-e^{-\rho_{2} R}$, and the corresponding PDF of the inter-vehicle distance which is smaller than the radio range can be expressed as:

$$
f_{2}(l \mid l<R)=F_{2}^{\prime}(l \mid l<R)=\frac{\rho_{2} e^{-\rho_{2} l}}{1-e^{-\rho_{2} R}} .
$$

Therefore, the probability that the link between $\mathrm{A} 1$ and $z_{w 1}$ is connected is:

$$
P_{A^{1} z_{w 1}}=P_{c}^{N_{A B}} .
$$

In addition, by substituting Equation (16) into (10), the PDF of $S_{N_{A B}}$ can be attained based on Equation (12) as:

$$
\begin{aligned}
f_{S_{N_{A B}}}(x) & =\frac{1}{2 \pi} \int_{-\infty}^{+\infty} e^{-j t x} \varphi_{S_{N_{A B}}}(t) d t, \quad x>0 \\
& =\rho_{2} e^{-\rho_{2} x} \frac{\left(\rho_{2} x\right)^{N_{A B}-1}}{\left(N_{A B}-1\right) !\left(1-e^{-\rho_{2} R}\right)^{N_{A B}}} .
\end{aligned}
$$

Then, the probability that $L_{A B}-R<S_{N_{A B}}<L_{A B}$ is:

$$
P_{S L}=\int_{L_{A B}-R}^{L_{A B}} f_{S_{N_{A B}}}(x) d x
$$

Based on Equation (17) and (19), the probability that the broken link between A and B in lane 1 can be connected with the assistance of the vehicles moving in the opposite direction can be expressed as:

$$
P_{A B}=P_{A^{1} z_{w 1}} P_{S L} .
$$

Given that there are $J$ broken links on lane 1, the connection probability of the whole road segment is: $P(J)=\prod_{J} P_{A B}$. Thereupon, according to Equation (15) and (20), the total connectivity of the road segment can be given by:

$$
p_{c p}=\left(P_{A B}\right)^{N_{g a p}^{1}} .
$$

3) Connection Quality: According to Equation (14) and (21), the connection quality of the road segment can be written as: $P_{c q}=P_{c p} P_{c d}(\alpha)$.

A case study is also investigated in this subsection as shown in Fig. 10 to reflect the impact of vehicular density and radio range on the connection quality. It can be concluded from Fig. 10 that there is close relationship among connection quality, density and radio transmission range.

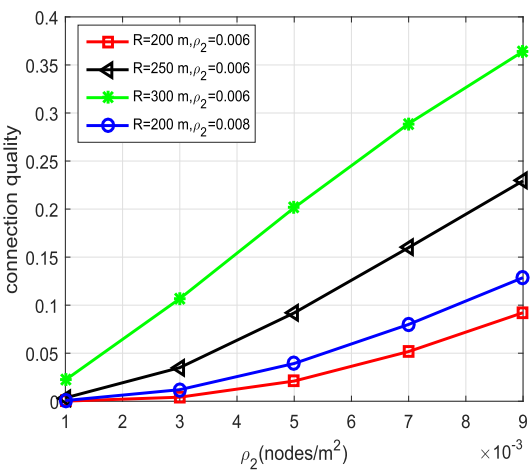

Fig. 10. A case study of the connection quality.

\section{B. The Delay Model}

Even with the connection distance into consideration, the connection quality still cannot completely reflect the transmission performance of a network. The transmission delay should be also taken into account as a critical parameter to measure the network performance [26].

In this subsection, we also give the following two cases to analyze the transmission delay, based on the relationship between the transmission range and the length of the road segment.

Case 1 (The Road Segment's Length Is Small Than R): When the length of the road segment is smaller than the transmission range, it is observed easily that the first vehicle in the road segment is with the transmission range of the last vehicle, thus the time needed for a packet from the first vehicle to the last one is called as one hop delay which includes the processing delay, the queuing delay, the link propagation delay, and the packet transmission delay [20]. For convenience, we assume the one hop delay is an average value. This assumption has been used by several work such as [10].

Case 2 (The Road Segment's Length Is Greater Than R): Without loss of generality, we first analyze the performance of the broken link between $\mathrm{A}$ and $\mathrm{B}$ on lane 1, and then give the model of the transmission delay of the whole road segment using the carry-and-forward scheme with the assistance of vehicles in the opposite lane.

Re-Healing Time: When the link between $\mathrm{A}$ and $\mathrm{B}$ is disconnected, packets from B can be sent to A through carryforward process, where the vehicles driving in the opposite lane can be utilized to bridge the communication gap between $\mathrm{A}$ and $\mathrm{B}$, restoring the link connection by temporal relay transmission. During this process, the time needed for data transmission is defined as the "re-healing time".

As shown in Fig.11, denote $x_{b z_{w 1}}$ as the distance between vehicle $z_{w 1}$ and B. Similarly, denote $x_{b z_{e 1}}$ as the distance between vehicle $z_{e}$ and $\mathrm{B}$. Let $C_{w}$ represent the platoon close to $z_{w 1}$, where each vehicle is within the transmission range of its adjacent vehicles, and $l_{c}$ is the length of the platoon.

This process can be divided into the following two sub-cases:

Sub-Case $1\left(x_{b z_{w 1}}<R\right)$ : If the inter-vehicle distance between $z_{w 1}$ and $c_{1}$ denoted by $x_{c_{1} z_{w 1}}$ is bigger than $R$, as shown in Fig.11, the packet from B needs to be carried by $z_{w 1}$ until reaching A's transmission range. The probability 


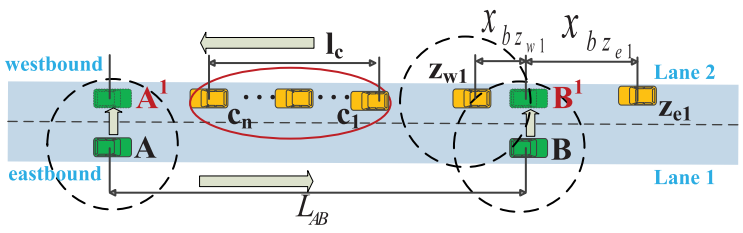

Fig. 11. A simplified example for sub-case 1.

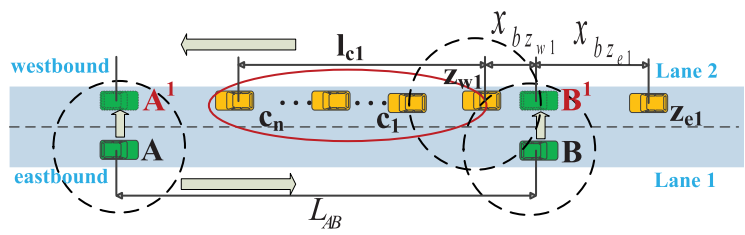

Fig. 12. Other simplified example for sub-case 1.

that this case happens is:

$$
\begin{aligned}
p_{1} & =\operatorname{Pr}\left\{x_{b z_{w 1}}<R\right\} \operatorname{Pr}\left\{x_{c_{1} z_{w 1}}>R\right\} \\
& =\left(1-e^{-\rho_{2} R}\right) e^{-\rho_{2} R} .
\end{aligned}
$$

In such a situation, the distance that the packet should traverse before reaching A's transmission range can be expressed as: $y_{1}\left(x_{b z_{w 1}}\right)=\max \left(0, L_{A B}-x_{b z_{w 1}}-R\right)$. As a result, the estimated time needed for a packet transmitted from B to A is:

$$
t_{1}=\int_{0}^{R} \frac{y_{1}(x)}{\hat{v}_{1}+\hat{v}_{2}} \frac{\rho_{2} e^{-\rho_{2} x}}{1-e^{-\rho_{2} R}} d x,
$$

Where $\hat{v}_{1}$ and $\hat{v}_{2}$ represent the average speed of vehicles in lane 1 and in lane 2, respectively.

If the inter-vehicle distance between $z_{w 1}$ and $c_{1}$ is smaller than $R$, as shown in Fig.12, the packet can be relayed faster with the assistance of the platoon $C_{w}$. The probability that this case happens is:

$$
\begin{aligned}
p_{2} & =\operatorname{Pr}\left\{x_{b z_{w 1}}<R\right\} \operatorname{Pr}\left\{x_{c_{1} z_{w 1}}<R\right\} \\
& =\left(1-e^{-\rho_{2} R}\right)^{2} .
\end{aligned}
$$

If $x_{b z_{w 1}}+l_{c}>L_{A B}-R$, the link between $\mathrm{A}$ and $\mathrm{B}$ is connected. Note that compared to the re-healing time taken for the restoration for a breakage link, which is on the order of seconds, the delay of a spatial relay between $\mathrm{A}$ and $\mathrm{B}$, which is on the order of milliseconds can be ignored. Otherwise, that is, $x_{b z_{w 1}}+l_{c}<L_{A B}-R$, the link between $\mathrm{A}$ and $\mathrm{B}$ is not connected, and the packet needs to be transmitted using the carry-and-forward mechanism. Then, the distance that the packet should traverse before reaching A's transmission range can be expressed as: $y_{2}\left(x_{b z_{w 1}}\right)=\max \left(0, L_{A B}-x_{b z_{w 1}}-l_{c}-R\right)$. To simplify the calculation, here $l_{c}$ is set as an average value of the platoon. As a result, the estimated time needed for a packet transmitted from B to A is:

$$
t_{2}=\int_{0}^{R} \frac{y_{2}(x)}{\hat{v}_{1}+\hat{v}_{2}} \frac{\rho_{2} e^{-\rho_{2} x}}{1-e^{-\rho_{2} R}} d x .
$$

Sub-Case $2\left(x_{b z_{w 1}}>R\right)$ : If there is no any vehicle between $\mathrm{B}$ and its west range on lane 2, as shown in Fig.13, then the broken link between A and B can only be restored by vehicles which are in the east of B. Then, the packet from

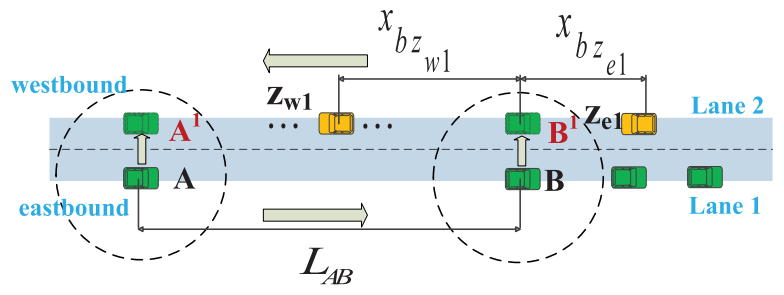

Fig. 13. A simplified example for sub-case 2 .

$\mathrm{B}$ needs to be relayed by $z_{e 1}$ in lane 2 to A. The probability that the case happens is:

$$
p_{3}=\operatorname{Pr}\left\{x_{b z_{w 1}}>R\right\}=e^{-\rho_{2} R} .
$$

In this case, the estimated time needed for a packet transmitted from $\mathrm{B}$ to $\mathrm{A}$ is:

$$
t_{3}=\int_{R}^{+\infty} \frac{\left(L_{A B}+x-R\right)}{\hat{v}_{1}+\hat{v}_{2}} \rho_{2} e^{-\rho_{2} x} d x .
$$

Based on the above analysis, the re-healing time during the carry-and-forward process can be given by :

$$
T_{c f}=p_{1} t_{1}+p_{2} t_{2}+p_{3} t_{3} .
$$

We use a set of simulations to verify the analysis of the re-healing time, as shown in Fig.14. It can be found that the analytical results are closely consistence with simulation results, which demonstrates the accuracy of the analytical model.

End-to-End Delay: Because of the network partitioning, a packet often traverse many gaps before it reaches the destination. Based on the memoryless property of the exponential distribution [27], it is known that all these gaps on the road are statistically independent which enables the individually analysis. So, Given that there are $J$ broken links on lane 1, the time needed for the packet through the whole road segment can be expressed as: $T(J)=\sum_{J} T_{c f}$. Thereupon, according to Equation (15) and (28), the total end-to-end delay of the road segment can be given by:

$$
T=N_{\text {gap }}^{1} T_{c f} .
$$

A case study is also investigated in this subsection as shown in Fig.15 to reflect the impact of vehicular density and speed on the end to end delay. It can be concluded from Fig.15 that there is close relationship among end to end delay, density and speed.

\section{Optimized Path Selection Algorithm}

Because of the mobility and distribution of nodes, there may be some partitioned islands of grouped vehicles along the routing path, which makes it difficult to transfer messages among vehicles. So the network connectivity becomes the main performance metric for inter-vehicle communications, particularly at a light load [25]. However, just utilizing the connectivity to select the route is not enough. As shown in Fig. 16, suppose the blue and black road paths have the same connectivity at the same time. According to the delay analysis above, if the black one has the lower delay compared with the 


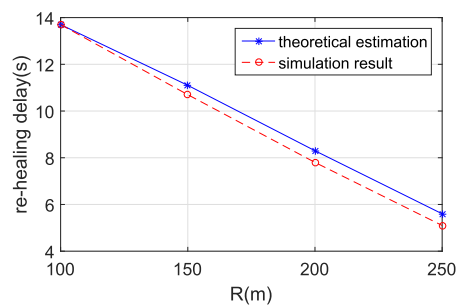

(a)

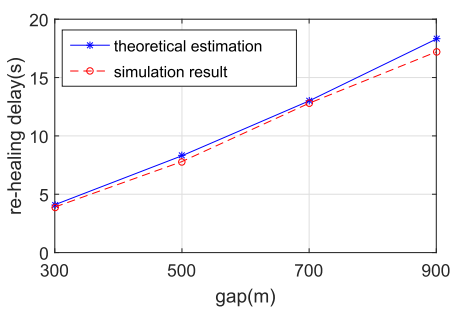

(b)

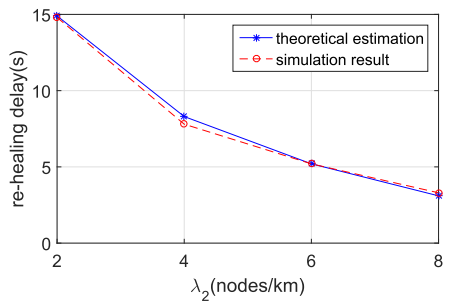

(c)

Fig. 14. Relationship between different parameters and re-healing time. (a) Relationship between transmission range $\mathrm{R}$ and re-healing time when gap $=500 \mathrm{~m}, \hat{v}_{1}=\hat{v}_{2}=20 \mathrm{~m} / \mathrm{s}, \rho_{1}=\rho_{2}=0.004 / \mathrm{m}$. (b) Relationship between broken link's gap and re-healing time when $R=200 \mathrm{~m}, \hat{v}_{1}=\hat{v}_{2}=20 \mathrm{~m} / \mathrm{s}$, $\rho_{1}=\rho_{2}=0.004 / \mathrm{m}$. (c) Relationship between vehicular density and re-healing time when gap $=500 \mathrm{~m}, R=200 \mathrm{~m} \hat{v}_{1}=\hat{v}_{2}=20 \mathrm{~m} / \mathrm{s}, \rho_{1}=0.004 / \mathrm{m}$.

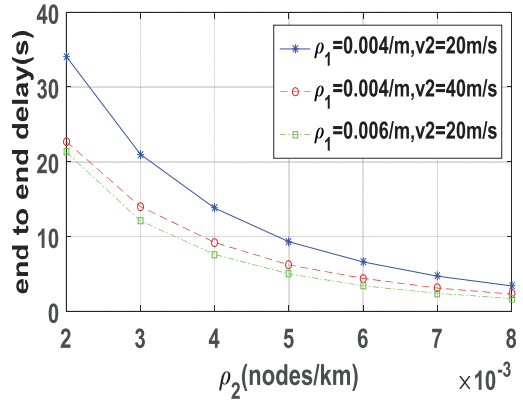

Fig. 15. A case study of the end to end delay when $L_{i}=1000 \mathrm{~m}, R=250 \mathrm{~m}$, $\hat{v}_{1}=20 \mathrm{~m} / \mathrm{s}$.

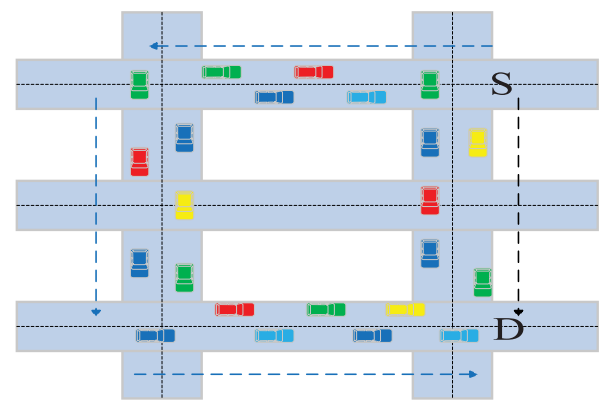

Fig. 16. Illustration for selecting a path based on the experienced delay.

blue one, it should have the high priority to be selected as a routing path. Therefore, besides the connectivity, the delay is also an important assistant factor for selecting a path between a source node and its destination node.

In light of the description above, we combine the path connection quality with the experienced delay to propose a new routing selection scheme as follows. Assume that there exist $n$ available backbone paths which are composed of different road segments from the source to the destination. Define $D_{r_{j}}$ as the delay needed for a data packet to its destination from the source along the road path $r_{j}(j=1,2 \cdots n)$ and $p_{r_{j}}$ as the corresponding road path connection quality. Given the fact that $r_{j}$ consists of $m$ road segments, and each road segment $r_{j i}(i=1,2 \cdots m)$ has an estimated transmission delay $d_{r_{j i}}$ and road segment connection quality $p_{r_{j i}}$, respectively. Then, the latency for a packet travelling through $r_{j}$ can be expressed as: $D_{r_{j}}=\sum_{i=1}^{m} d_{r_{j i}}$. Similarly, we can get the connection quality of path $r_{j}: p_{r_{j}}=\prod_{i=1}^{m} p_{r_{j i}}$. We select the road path $r_{\text {optimal }}$ as the optimized path whose connection quality is the highest among all the available paths. Then $r_{\text {optimal }}$ can be deduced as:

$$
r_{\text {optimal }}=\arg \min _{j \in(1,2, \cdots, n)}\left(p_{r_{j}}\right) .
$$

When there are more than one road paths, e.g. $k$, with the similar connection quality higher than other road paths, let define $U$ as the set of the $k$ paths. Further, we can select the one with the lowest delay within $U$ as the optimized path, i.e.,

$$
r_{\text {optimal }}=\arg \min _{j \in U}\left(D_{r_{j}}\right) .
$$

\section{PAcket Forwarding Procedure}

Upon the optimized path has been selected, the sent packets will be forwarded along this path from the source to the destination node. Thus, it is quite important to select the next hop to relay the packet. Next, we first construct the set of available neighbors which satisfy some given requirements, and then propose the proposed selective forwarding scheme.

\section{A. Construct the Set of Available Neighbors}

Step 1: Due to the high mobility of vehicles, to avoid outof-date neighbors, we first need to predict the positions of one node's neighbors. Denote $i$ be one sender, and $j$ be its receiver. $j$ reported its coordinate position $\left(x_{j}\left(t_{0}\right), y_{j}\left(t_{0}\right)\right)$ and speed vector $\left(v_{j}, \theta_{j}\right)$ to $i$ from last beacon at the moment $t_{0}$. Then, at time $t$ before exchanging the information again through the next beacon, $i$ can estimate $j^{\prime} s$ coordinate as follows:

$$
\left\{\begin{array}{l}
x_{j}{ }^{\prime}(t)=x_{j}\left(t_{0}\right)+v_{j} \operatorname{con}\left(\theta_{j}\right)\left(t-t_{0}\right) \\
y_{j}{ }^{\prime}(t)=y_{j}\left(t_{0}\right)+v_{j} \sin \left(\theta_{j}\right)\left(t-t_{0}\right) .
\end{array}\right.
$$

Actually, only those still within the radio range of $i$ are considered as the candidates for the next hop selection, namely: $d_{i j}=\sqrt{\left(x_{j}{ }^{\prime}(t)-x_{i}(t)\right)^{2}+\left(y_{j}{ }^{\prime}(t)-y_{i}(t)\right)^{2}} \leq R$, where $\left(x_{i}(t), y_{i}(t)\right)$ is the coordinate of $i$ at time $t$, and $\left(v_{i}, \theta_{i}\right)$ is its speed. Those nodes which meet the above condition will form a set $U_{i}{ }^{1}$.

Step 2: In VANETs, there are many moving and static objects to reflect, scatter, diffract or even block the signals. In order to the successful reception of a packet, the transmission probability calculated by Equation (1) between $i$ and $j$ should be greater that one certain threshold $p_{t h}^{f}$ [28]. Then we can further get a new set $U_{i}{ }^{2}$ composed of those nodes in $U_{i}{ }^{1}$ which meet the condition. 


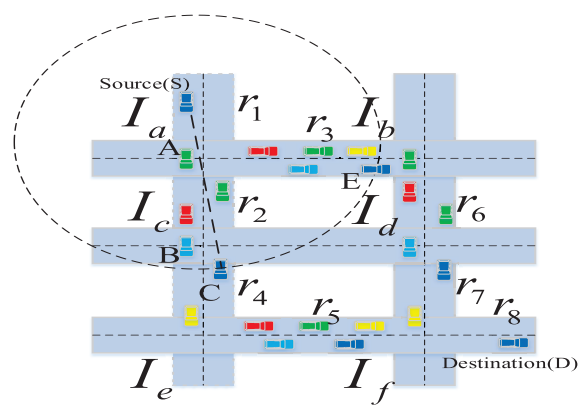

Fig. 17. Illustration for selective forwarding.

Step 3: Denote $T_{i j}$ as the time needed to successfully complete a packet from $i$ to $j$. If there are $k$ packets in the buffer when a new packet arrives to the queue, $T_{i j}$ can be further expressed as [20]: $T_{i j}=T_{t}+T_{q}=(k+1) T_{t}$, where $T_{t}$ stands for the transmission delay and $T_{q}$ represents the queuing time. For the purpose of guaranteeing the successful transmission between $i$ and $j$, the link connection time $l i n k_{i j}$ between them calculated by Equation (5) should be bigger than $T_{i j}$. We further select the available neighbors from $U_{i}^{2}$ which meet the requirement to form a new set $U_{i}{ }^{3}$.

Through these steps above, we get construct one set of available neighbors, from which one node can be selected as the next hop according to its priority determined by the relay strategy.

\section{B. Selective Forwarding Scheme}

We define the node located on the intersection as the junction node and the node placed within the road segment as the simple node. When one node receives a packet, it first checks if the destination node is in its radio range; if so, the packet is directly forwarded to the destination node. Otherwise, we follow the forwarding method described below.

1) There Are No Available Junction Nodes in Its Neighbor List: It selects the node closest to the next junction of the road segment from its set of available neighbors. Then, the optimal next hop $h_{o p t}$ can be selected using the following criterion: $h_{o p t}=\min _{j \in U_{s}} d(j, I)$, where $U_{s}$ is the set of the sender's available neighbors, $d(j, I)$ indicates the distance from one node $j$ in the set to intersection $I$, which can be given by: $d(j, I)=\sqrt{\left(x_{j}-x_{I}\right)^{2}+\left(y_{j}-y_{I}\right)^{2}} .\left(x_{j}, y_{j}\right)$ and $\left(x_{I}, y_{I}\right)$ represent the coordinate positions of node $j$ and intersection $I$, respectively.

2) There Are Some Junction Neighbor Nodes in Its Neighbor List: It first checks whether the subsequent road segments in the optimized routing path are an extension of the current road segment. If so, these road segments can be equivalent to one virtual road segment (VRS). The packet can be directly forward to the node closest to the farthest junction in the set $U_{s}$ along the VRS. By this way, the average hop count of the route can be reduced. Otherwise, the packet is forwarded to the neighbor junction node. As shown in Fig. 17, the optimized routing path is composed of some road segments between $\mathrm{S}$ and $\mathrm{D}$, i.e., $r_{1} \rightarrow r_{2} \rightarrow r_{4} \rightarrow r_{5} \rightarrow r_{8}$. When $\mathrm{S}$ wants to forward the packet, it is found that the junction node A, B is with its radio range. Based on the traditional junction node first

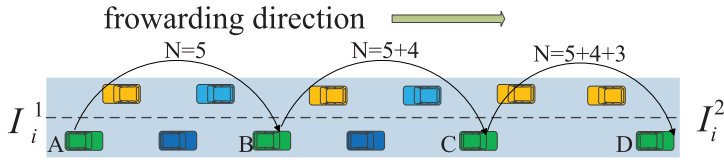

Fig. 18. On-the-fly density collection mechanism.

policy, S will select B as the next hop, and then B will select $\mathrm{C}$ as the relay. Thus, two hops are needed. Different from the traditional method, since $r_{2}$ and $r_{4}$ are the extension of $r_{1}$, we can equalize $r_{1} \rightarrow r_{2} \rightarrow r_{4}$ to be one VRS $r_{124}$.Thus, the data from $\mathrm{S}$ can be directly to the node $\mathrm{C}$, reducing the hop number. However, if the optimized routing path is $r_{1} \rightarrow r_{3} \rightarrow r_{6} \rightarrow r_{7} \rightarrow r_{8}$, since the junction node $\mathrm{A}$ is within the S's range but the next road section $r_{3}$ is not an extension of $r_{1}$, the data is thus directly forwarded to A.

3) There Exists No Neighbor Node Which Is Closer to the Junction Than Itself: We adopt the carry-and-forward scheme to forward the data packets.

\section{Protocol Optimization Scheme}

\section{A. Road Information Updating}

Note that our work is based on the assumption that vehicles are equipped with GPS and the electronic map. In this way, vehicles can know not only the land attributes such as the road topology and the locations of the intersections, but also the traffic statistics such as traffic density and average speed within an area at a certain time in a day. If the obtained information of each road segment is precise, we can correctly work out the path connection quality and forwarding delay by which to select an optimized path between the source and destination pair. However, in practice, there may be some errors in the collected statistical data. As a result, the on-the-fly density collection is adopted in our paper to update the statistics, as illustrated in Fig. 18, where A is the forwarding node and B is the next hop. By periodically exchanging HELLO packets with the one hop neighbors, A can acquire the information (e.g. position and speed) of its neighbors. Then, A adds the number of its neighbors (e.g. 5) and the sum of their speeds to the packet header and forwards it to B. Next, B follows the same procedure until the packets are forwarded to $\mathrm{D}$ at the end of the road segment. Finally, the density and the average speed of the vehicles on the road segment can be collected.

\section{B. Position Tracking Mechanism}

Because of the mobility, before receiving data packets sent from the source, there is the chance that the destination node has moved far way from its original location. Particularly, in case of the sparse environment, the carry-and-forward scheme should be employed, further increasing the probability of the destination node moving far way. Thus, the position tracking scheme is designed to deal with this issue. When the destination is leaving the intersection, it attaches its velocity vector, namely, the speed and the direction of its movement, which can indicate where it is intended to go, to the next beacon it broadcasts. All the nodes which hear the beacon will keep the information as long as they reside at that intersection and rebroadcast it before they are about to leave the 
TABLE I

Network Performance Evaluation Parameters

\begin{tabular}{l|l}
\hline Description & Value \\
Beacon message size & 20 bytes \\
Maximum transmission range & $300 \mathrm{~m}$ \\
Beacon period & $3 \mathrm{~s}$ \\
Mac protocol & $802.11 \mathrm{p}$ \\
BROADCAST_TIMER & $1 \mathrm{~s}$ \\
Interface queue & PriQueue \\
Interface queue length & 20 \\
Packets generation speed & $1-10$ packets/s \\
Packet size & 512 bytes \\
Channel capacity & $3 \mathrm{Mbps}$ \\
\hline
\end{tabular}

TABLE II

SCENARIO GENERATION PARAMETERS FOR IDM_LC

\begin{tabular}{l|l}
\hline Description & Value \\
Simulation area & $2000 \mathrm{~m} * 1500 \mathrm{~m}$ \\
Number of vehicles & 200 \\
Traffic-light interval & $10 \mathrm{~s}$ \\
Number of lanes & 2 \\
Minspeed & $6.66 \mathrm{~m} / \mathrm{s}$ \\
Maxspeed & $24.44 \mathrm{~m} / \mathrm{s}$ \\
recalculating movement step & $1 \mathrm{~s}$ \\
Minstay-Maxstay & $5-30 \mathrm{~s}$ \\
\hline
\end{tabular}

intersection. When one sent packet from the source reaches the intersection, its carrier along the path can get the information where the destination is moving and then forwards the packet to the destination. Besides, with the help of GPS and location services, when finding that the destination node has moved out of its original road segment, the source node will initiate one new route selection procedure.

\section{Performance Simulation}

In this section, our proposed ASGR protocol is implemented on a vehicular communication test-bed combing Matlab and NS2 on Linux platform. Its performance is compared with three protocols, namely, ACAR [9], MAR-DYMO [18] and HyBR [5], where ACAR is a connectivity-aware routing protocol, MAR-DYMO and HyBR are both bio-inspired routing protocols.

\section{A. Simulation Scenario}

The street layouts used for our simulations are loaded from the TIGER database. The movement profile of vehicles was generated by the VanetMobiSim [29] toolkit using IDM_LC (intelligent driver model with lane changing) mobility model [29]. The experiment is restricted in a $2000 \mathrm{~m} * 1500 \mathrm{~m}$ rectangle area. The data streams of source nodes are generated with constant bit rate (CBR). The simulation parameters and mobility configurations are listed in TABLE I and II, respectively. Fig. 19 and Fig. 20 are satellite snapshots of selected area in Washington D.C. and Favellas in Rio, respectively. The usage of different maps is to reflect our model's scalability and alleviate the specificity of results from an individual map.

In addition, although TIGER can describe land attributes such as roads, buildings, rivers, and lakes, it is still difficult to draw obstacles on output traces by VanetMobiSim. As a result, to reflect the impact of obstacles on signal attenuation involving buildings and moving vehicles, we extract the

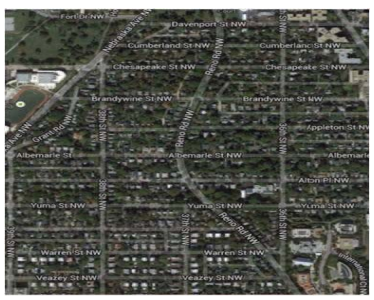

Fig. 19. Selected area of Washing-ton D.C.

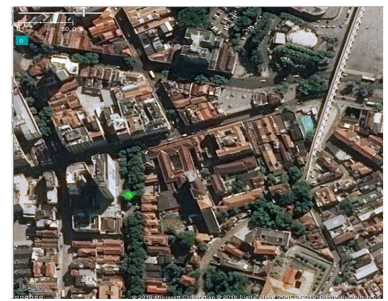

Fig. 20. Selected area of Favellas in Rio.

coordinates of obstacles from the investigated maps and input them into NS2. Besides, since there is no height information in TIGER database, a modification to NS2 is needed to reflect the impact of obstacles on channel fading and power attenuation. To support obstacle modeling, a two-dimension obstacle object "ObstacleClass" is introduced which represents a wall of 1 meter deep and has the length indicated by the distance between two coordinates extracted from the real maps, i.e. $P_{1}\left(x_{1}, y_{1}\right)$ and $P_{2}\left(x_{2}, y_{2}\right)$. By this way, a building could be expressed by four connected walls. To reflect the blocking of signals by moving vehicles, we model all the vehicles with the same size, i.e. $5 \mathrm{~m}$ (length) $* 2 \mathrm{~m}$ (width) with four surfaces of 0.1 meter deep. When the Line Of Sight(LOS) of a communication pair intersects with the outline of the obstacles, the power attenuation could be calculated by the following equation, which combines the Nakagami-m radio propagation model with the obstacle model presented in [30], i.e.

$$
P_{r}[d B m]=P_{t}[d B m]+10 \lg \left(\frac{G_{t} G_{r} \lambda^{2}}{16 \pi^{2} d^{\alpha}}\right)-\beta n-\gamma d_{m},
$$

where $P_{r}, P_{t}, G_{t}, G_{r}, \lambda, d$ are the receive power, transmit power, sender antenna gain, receiver antenna gain, wavelength and the distance between sender and receiver, respectively. $n$ is the number of times that the border of the obstacle is intersected by the line of sight. $d_{m}$ here is the total length of the obstacle's intersection. $\beta$ and $\gamma$ are two constants. $\beta$ is given in $\mathrm{dB}$ per wall/surface and represents the attenuation a transmission experiences due to the exterior wall of a building or surface of a vehicle. $\gamma$ is given in $\mathrm{dB}$ per meter and serves as a rough approximation of the internal structure of a building or a vehicle. The general values of $\beta$ and $\gamma$ in most cases are $9 \mathrm{~dB}$ and $0.4 \mathrm{~dB} / \mathrm{m}$ respectively. Finally, each scenario is repeated 50 times to achieve the arithmetic mean.

\section{B. Performance Metrics}

Average Transmission Delay (ATD): defined as the average difference between the time a data packet is originated by an application and the time this packet is received at its destination. 


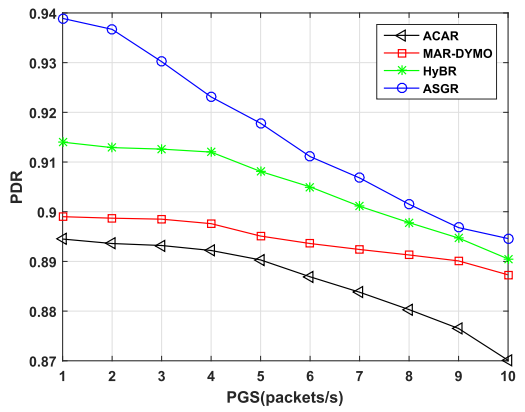

Fig. 21. PDR vs. PGS for four protocols in Washington D.C.

Packet Delivery Ratio (PDR): defined as the number of correctly received packets at the destination vehicle over the number of packets sent by the source vehicle.

Routing Overheads (RO): defined as the ratio between the total number of bytes of control packets and the cumulative size of data packets delivered to the destinations.

\section{Numerical Results and Corresponding Analysis}

In this subsection, we fist give the detailed analysis of the impact of varying Packets Generation Speed (PGS) and Number of Vehicles (NV) on the network performance through a series of simulations, where one source node is randomly selected to communicate with a randomly selected moving destination. It is noticed that the listed number of vehicles in TABLE II, i.e. 200, is just applied to the specific scenarios for PGS changing. Correspondingly, the used packet generation speed remains at 5 packets/s when the NV changes. After that, we also analyze the impact of the number of CBR connections on the network performance, where different pairs of source and destination nodes are randomly selected to exchange data packets. Note that to reflect our model's scalability and alleviate the specificity of results from an individual map, the performance of four protocols is compared in two different maps with different complexity and configurations on the number of junctions and streets, and average length of streets. Here, the map of selected area in Washington D.C. is gridding while the map of selected area in Favellas in Rio is relatively irregular. Finally, we only show part of the results under two maps considering the limitation of space and number of figures.

1) The Impact of Varying PGS on the Network Performance: The PDR performance is evaluated with the variation of PGS in Fig. 21. Basically, the increase of PGS will slightly decrease the PDR for all protocols. This is because that the fewer the packets generated per second on the sender, the smaller the probability that a packet is dropped due to channel failure, buffer overflow or delay deadline miss. Note that a packet may be send out but not successfully received considering the medium access collision and channel fading especially when the impact of obstacles on signal attenuation is taken into account. In addition, before a route to the destination has been obtained, the continuously generated packets might make the sending buffer overflow especially in the case of store-carry forwarding. Also, there might be some packets still in buffers till the end of the simulation and these packets will be dropped due to their huge delays. From Fig. 21, ASGR achieves the highest PDR. Actually, since connection quality and expected latency are considered during the route selection, ASGR could make the PDR maximized. Besides, because multiple paths routing and store-carry forwarding are enabled, a packet will be transmitted to its destination with best efforts.

Next, it can be noted that HyBR and MAR-DYMO give the second and third highest PDR, respectively. Since HyBR utilizes a reactive topology-based routing scheme when density is high and a geography-based routing scheme when density is low, its PDR shows better than MAR-DYMO at a cost of more introduced overheads and computation complexity in this 200-vehicle scenario. Since Bitam et al. [5] indicated that the best configuration of the density determining threshold is 50 vehicles when the radio range is set to 300 meters (as listed in TABLE I), we also apply this value to our discussed scenario. According to this threshold, HyBR may execute the topology-based routing in some areas requiring that each node saves the position information of all nodes in a table called a positions table which is updated whenever the network topology changes. In this way, the network topology could be accurately reflected in a real time manner thus leading to a high PDR. It is also worth noting that similar to our ASGR, HyBR also takes the delay incurred to reach the destination into account when selecting a path. The performance difference between ASGR and HyBR, from my perspective, is due to the consideration of catch-up and carry-forward mechanisms in our scheme which utilizes the "indirect connectivity" to deliver the packets instead of just dropping it when a broken link is detected.

MAR-DYMO is also a reactive bio-inspired routing protocol but with mobility awareness. Whenever a difference between the actual and predicted position is larger than a predefined threshold, a new broadcast is performed to update the position information stored in each node. Although a node with MAR-DYMO could timely response to the position change of its neighbors, the multiple paths finding depending on the global knowledge between two nodes still needs a nontrivial time period and thus is not a real-time process. In this way, MAR-DYMO may adopt some globally outdated paths as the routing paths determined by the ACO algorithm and make some packets dropped when the next hop is not reachable.

As for ACAR, it is a geography-based routing protocol. And similar to ASGR, it also uses the transmission quality of a path to select the route. The transmission quality defined in ACAR is a product of the delivery ratio and probability of the network connectivity for a certain route, which relies on accurate network density collection and statistical PER (Packets Error Rate) of the entire route. These assistant data are collected through the periodical beacons sent by each node. As shown in Fig. 21, ACAR shows the worst PDR compared to others. By exploring the NS2 trace file, it can be found that there are mainly three reasons resulting in the performance degradation. The first one is from the used channel model which has taken the NLOS case into consideration. Since we import many obstacles on the map, ACAR sometimes cannot find an available next hop thus needing another route discovery procedure. In addition, as listed in TABLE I, we set the beacon interval to 3 seconds to reduce the overhead and network burden. However, ACAR is a protocol which strongly relies 


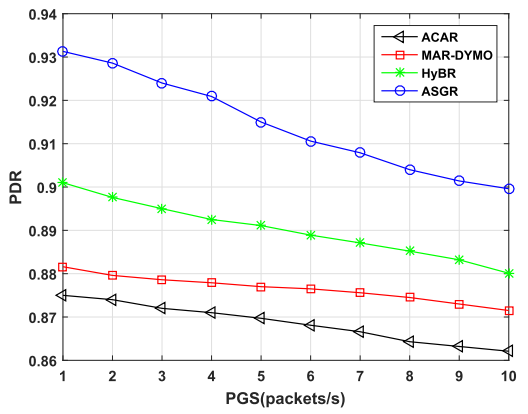

Fig. 22. PDR vs. PGS for four protocols in Favellas in Rio.

on the beacon's up-to-datedness. Compared to the 1-second configuration in [9], a 3-second setting may make the global information of ACAR outdated. Besides, compared to the other three protocols which are all multiple path routing algorithms, ACAR can only return back one choice to the source node which will fail the ongoing transmission when there is no available path to the destination.

To alleviate the impact of a specific map on the test results, we also run the PDR simulation on the map of selected area in Favellas in Rio, Brazil with the irregular non-grid topology, as shown in Fig. 22. Compared with Washington D.C., the considered area of Favellas in Rio has more complexity in terms of more streets, junctions and shorter average length of streets. What is more, in the considered area, road segments are vary narrow and resemble spider web. Note that the rank of the PDR performance of four protocols is the same as shown in Fig. 21. However, through careful checking and comparing of the points between Fig. 21 and Fig. 22, it can be founded that MAR-DYMO and ACAR both show lower PDR during the entire simulation process in Fig. 22 compared to that in Fig. 21. This is because the complexity of the map of Favellas in Rio makes MAR-DYMO generate more periodic HELLO messages to reactively search for more available paths to the destination. As for ACAR, the per-segment based PER and connectivity collection process will need more assistant data from the periodical beacons due to more complexity of introduced map. Instead, ASGR outputs a better PDR when PGS is 10 but generates a worse PDR when PGS is only 1 compared to Fig. 21 . This result indicates that the introduce overhead of our ASGR is non-correlated with the map complexity since proactive routing is enabled by which the link quality information has been collected in advance by the spiders rather than been obtained on demand. In addition, since overhead is almost fixed for ASGR, the fewer the PGS, the more the ratio of the routing overhead to the sent traffic. This conclusion is also verified in Fig. 24. The HyBR starts at a lower PDR in Fig. 22 when PGS is 1 but consequently outputs the same points in both figures when PGS is 10. Actually, since HyBR employs topology-based reactive routing scheme when the network is highly dense, its performance is sensitive to the map complexity because more routing overheads have been generated.

The ATD performance of four protocols has been compared as shown in Fig. 23 and Fig. 24, respectively. Same as Fig. 21 and Fig. 22, the ATD shows the same rank among four protocols. This result is reasonable since more PDR will

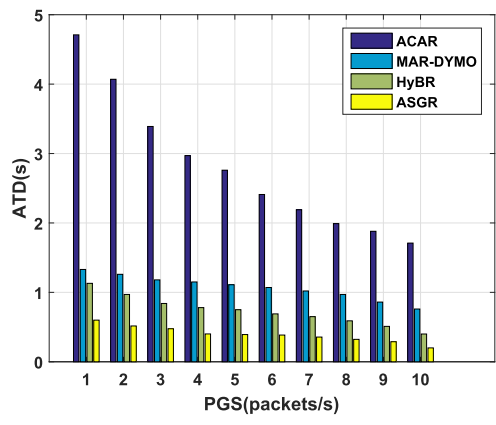

Fig. 23. ATD vs. PGS for four protocols in Washington D.C.

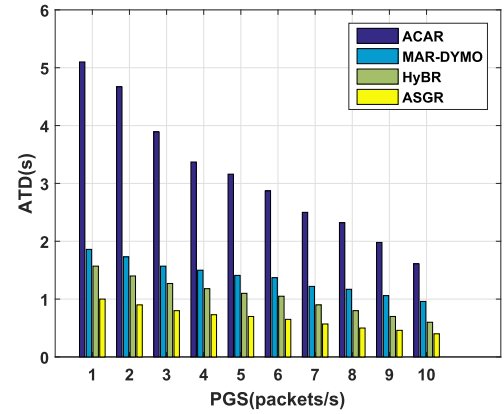

Fig. 24. ATD vs. PGS for four protocols in Favellas in Rio.

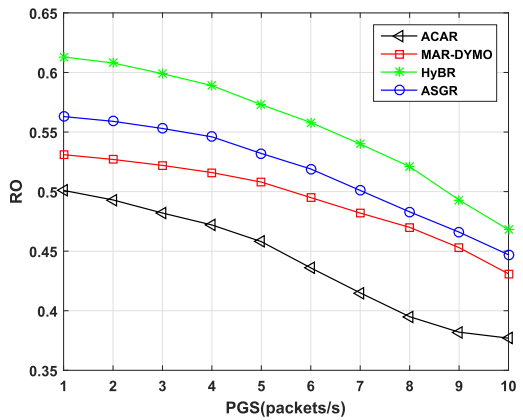

Fig. 25. RO vs. PGS for four protocols in Washington D.C.

correspondingly generate lower average transmission delay due to few retransmitted packets and latency extension caused by channel failure, buffer overflow or delay deadline miss. Additionally, since our ASGR takes the expected latency into consideration, its performance even meets the delay requirement for the specified related events according to TABLE I when the PGS is 5. Compared with Fig. 23, it is found that Fig. 24 shows higher ATD during the entire simulation process. This is because in the irregular topology, one determined route will include more intersections, thus leading to the increase of the number of hops needed to forward the packet and incurring the longer delay.

The RO of four protocols has been checked with results depicted in Fig. 25. Note that since ACAR is the only one which does not utilize the bio-inspired idea in the routing process, its RO shows the smallest among the four protocols. This is reasonable because there are no proactive routing overheads used in ACAR thus leading to a lower RO at a price of poor PDR performance. Even the statistical density information for road segment selection is collected on-the-fly while forwarding packets, which will not make the overheads increase significantly. As for the comparison of RO among 


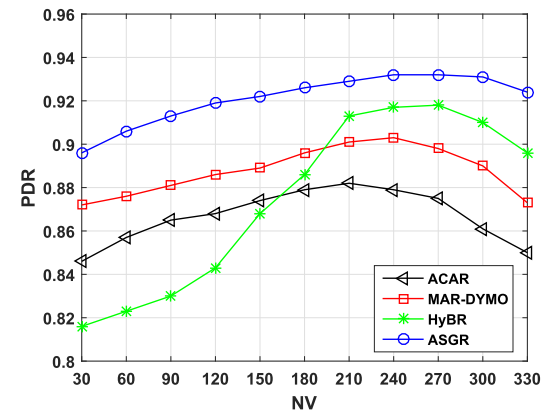

Fig. 26. PDR vs. NV for four protocols in Washington D.C.

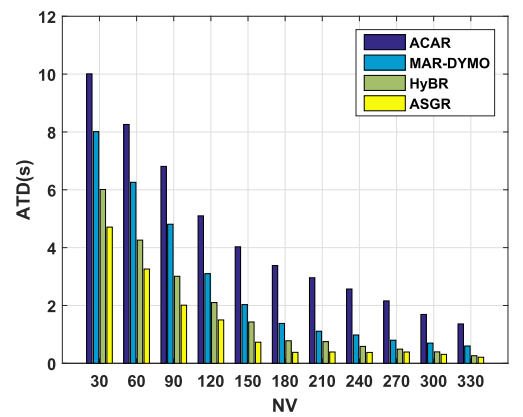

Fig. 27. ATD vs. NV for four protocols in Washington D.C.

the three bio-inspired algorithms, our ASGR shows a decent performance in between HyBR and MAR-DYMO. Because HyBR employs the topology-based routing scheme in this scenario, its $\mathrm{RO}$ is the highest considering the frequently issued beacons and proactively used bees for topology detecting and multi-path searching. The MAR-DYMO shows a satisfied RO in this case due to its reactive property during routing discovery. Although MAR-DYMO does not need the proactively topology information which may incur large routing overhead, its reactively routing searching also introduces more overhead than ACAR considering its usage of ant-colony and periodic HELLO messages.

2) The Impact of Varying NV on the Network Performance: Next, we begin to explore the impact of NV on the performance of four protocols with results depicted in the Fig. 26 and Fig. 27. Different from Fig. 21 and Fig. 22, the PDR of four protocols for this case shows different rank as shown in Fig. 26. In general, with the increasing of NV, all protocols show rising PDR first but then experience dropping with different extent. The reason behind is that more vehicles have also brought greater possibility for packet collisions and buffer overflow. In addition, the topology changing is also drastic under high density environments, which will frequently initiate the routing discovery procedure thus increasing the overheads and correspondingly reducing the ratio of successfully received payloads. Among the four algorithms, HyBR shows the most fluctuant PDR with an amplitude ranging up to almost $10 \%$. By checking the simulation trace, it can be found that HyBR has changed its routing strategy during the test according to the varying of the vehicular density. In other words, HyBR first executes a geography-based routing when the density is low but then switches to the topology-based routing where there are too many vehicles, depending on the predefined threshold in [5].

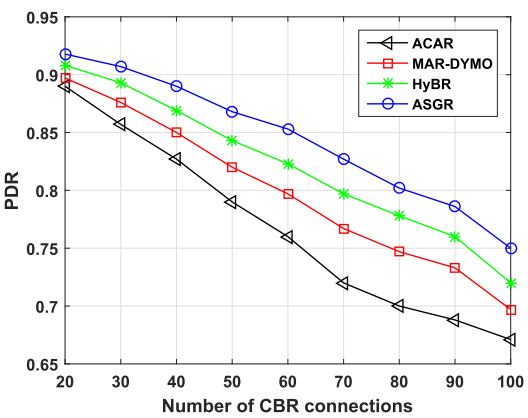

Fig. 28. PDR vs Number of CBR connections in Washington D.C.

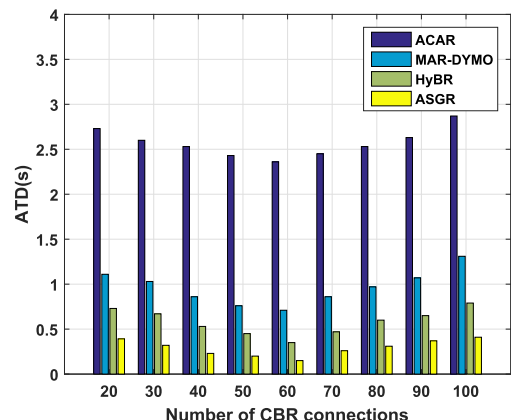

Fig. 29. ATD vs Number of CBR connections in Washington D.C.

The ATD performance of four protocols is evaluated in Fig. 27. Similar with Fig. 23, the ACAR shows the worst ATD. Note that ACAR seems to be very sensitive to the varying of vehicular density so that its ATD shows a sustained growing with an increasing growth rate. In other words, ACAR may generate intolerable delay for vehicular applications when the density is ultra-high. Actually, by exploring the simulation trace of ACAR, it can be found that there are more packets suffered from network disconnection and longer waiting time of buffering, attributed to the inaccurate density estimation by the real-time on-the-fly density collection process. The other three protocols all show decent ATD and our ASGR performs the best.

3) The Impact of Varying CBR Connections on the Network Performance: Fig. 28 indicates the performance of PDR as the function of the number of CBR connections. From the figure, it is found that with the increase of the number of CBR connections, the PDR of all four routing protocols are decreasing. The reason is that when the number of CBR connections is increased, the probability of data packets losses due to buffer overflow and channel contention is growing. Compared with other three routing protocols, ASGR achieves the best PDR. This is because ASGR aims to improve the connection probability of between vehicles, which can guarantee the sent packet successfully reaches the destination as soon as possible before it expires.

Fig. 29 describes the ATD with the number of CBR connections varying. When the number of CBR connections is increased, the ATD mainly depends on two factors. 1) The data packet losses caused by the buffer overflow make the number of packets stored and carried decrease as well as the average transmission delay. In other words, there are few packets needed to be carry-and-forward thus making the average transmission delay decrease. 2) The queuing delay in 
the buffer is increased with the growing of the number of total transmitted packets. When the number of CBR connections is smaller than 60 in our simulations, the former is the leading factor. Therefore, the ATD of all four routing protocols is first decreased and finally increased with the number of CBR connection varying. Actually, since our ASGR selects the routing path with the maximum connection quality as the best route, which can reduce the use of the carry-and-forward scheme to a great extent, thus exhibiting the minimum delay compared with other routing protocols.

\section{Conclusion}

The ASGR protocol, designed to optimally route the data packets using the artificial spider-web technology in urban environments, efficiently considers the characteristics of road traffic, urban topology and geographic information to intelligently serve for the applications in VANETs. First, the available paths between the source and destination node are determined through the use of artificial spiders and with the help of the available digital map. In this way, the communication overheads could be greatly reduced. Then, we take path connectivity, delay into consideration to improve the route selection mechanism. When one optimized route is selected, the proposed selective forwarding scheme is executed to forward the packet. Simulation results show that ASGR performs better in terms of end-to-end delay and packet delivery ratio. It should be able to provide stable communication while maintaining higher delivery ratio and lower delay for vehicular routing in urban environments.

\section{REFERENCES}

[1] K. Zheng, Q. Zheng, P. Chatzimisios, W. Xiang, and Y. Zhou, "Heterogeneous vehicular networking: A survey on architecture, challenges, and solutions," IEEE Commun. Surveys Tuts., vol. 17, no. 4, pp. 2377-2396, 4th Quart., 2015.

[2] N. Alsharif and X. Shen, "iCAR-II: Infrastructure-based connectivity aware routing in vehicular networks," IEEE Trans. Veh. Technol., vol. 66, no. 5, pp. 4231-4244, May 2017.

[3] C. Chen, Y. Jin, Q. Pei, and N. Zhang, "A connectivity-aware intersection-based routing in VANETs," EURASIP J. Wireless Commun. Netw., vol. 2014, no. 1, pp. 1-16, 2014.

[4] A. Kaul, K. Obraczka, M. A. S. Santos, C. Rothenberg, and T. Turletti, "Dynamically distributed network control for message dissemination in ITS," in Proc. IEEE/ACM DS-RT, Oct. 2017, pp. 1-9.

[5] S. Bitam, A. Mellouk, and S. Zeadally, "HyBR: A hybrid bio-inspired bee swarm routing protocol for safety applications in vehicular ad hoc networks (VANETs)," J. Syst. Archit., vol. 59, no. 10, pp. 953-967, 2013.

[6] S. Bitam, A. Mellouk, and S. Zeadally, "Bio-inspired routing algorithms survey for vehicular ad hoc networks," IEEE Commun. Surveys Tuts. vol. 17, no. 2, pp. 843-867, 2nd Quart., 2015.

[7] V. Naumov, R. Baumann, and T. Gross, "An evaluation of inter-vehicle Ad hoc networks based on realistic vehicular traces," in Proc. ACM MOBIHOC, 2006, pp. 108-119.

[8] V. Naumov and T. R. Gross, "Connectivity-aware routing (CAR) in vehicular Ad-hoc networks," in Proc. IEEE INFOCOM, May 2007, pp. 1919-1927.

[9] Q. Yang, A. Lim, S. Li, J. Fang, and P. Agrawal, "ACAR: Adaptive connectivity aware routing for vehicular ad hoc networks in city scenarios," Mobile Netw. Appl., vol. 15, no. 1, pp. 36-60, Feb. 2010.

[10] H. Saleet, R. Langar, K. Naik, R. Boutaba, A. Nayak, and N. Goel, "Intersection-based geographical routing protocol for VANETs: A proposal and analysis," IEEE Trans. Veh. Technol., vol. 60, no. 9, pp. 4560-4574, Nov. 2011.

[11] S. Tsiachris, G. Koltsidas, and F.-N. Pavlidou, "Junction-based geographic routing algorithm for vehicular ad hoc networks," Wireless Pers. Commun., vol. 71, no. 2, pp. 955-973, 2013.
[12] P. Sermpezis, G. Koltsidas, and F. N. Pavlidou, "Investigating a junctionbased multipath source routing algorithm for VANETs," IEEE Commun Lett., vol. 17, no. 3, pp. 600-603, Mar. 2013.

[13] K. Shafiee and V. C. M. Leung, "Connectivity-aware minimum-delay geographic routing with vehicle tracking in VANETs," Ad Hoc Netw. vol. 9, no. 2, pp. 131-141, 2011.

[14] M. A. Togou, A. Hafid, and L. Khoukhi, "A novel CDS-based routing protocol for vehicular ad hoc networks in urban environments," in Proc. IEEE GLOBECOM, Dec. 2015, pp. 1-6.

[15] M. A. Togou, A. Hafid, and L. Khoukhi, "SCRP: Stable CDS-based routing protocol for urban vehicular ad hoc networks," IEEE Trans. Intell. Transp. Syst., vol. 17, no. 5, pp. 1298-1307, May 2016.

[16] J. Rak, "LLA: A new anypath routing scheme providing long path lifetime in VANETs," IEEE Commun. Lett., vol. 18, no. 2, pp. 281-284, Feb. 2014.

[17] X. Zhang, X. Cao, L. Yan, and D. K. Sung, "A street-centric opportunistic routing protocol based on link correlation for urban vanets," IEEE Trans. Mobile Comput., vol. 15, no. 7, pp. 1586-1599, Jul. 2016.

[18] S. L. O. Correia, J. Celestino, and O. Cherkaoui, "Mobility-aware ant colony optimization routing for vehicular ad hoc networks," in Proc. IEEE WCNC, Mar. 2011, pp. 1125-1130.

[19] H. Saleet, O. Basir, R. Langar, and R. Boutaba, "Region-based locationservice-management protocol for VANETs," IEEE Trans. Veh. Technol., vol. 59, no. 2, pp. 917-931, Feb. 2010

[20] P. K. Sahu, E. H.-K. Wu, J. Sahoo, and M. Gerla, "BAHG: Backbone-assisted hop greedy routing for VANET's city environments," IEEE Trans. Intell. Transp. Syst., vol. 14, no. 1, pp. 199-213, Mar. 2013.

[21] A. B. Reis, S. Sargento, F. Neves, and O. K. Tonguz, "Deploying roadside units in sparse vehicular networks: What really works and what does not," IEEE Trans. Veh. Technol., vol. 63, no. 6, pp. 2794-2806, Jul. 2014.

[22] M. H. Eiza, T. Owens, and Q. Ni, "Secure and robust multi-constrained QoS aware routing algorithm for VANETs," IEEE Trans. Depend. Sec. Comput., vol. 13, no. 1, pp. 32-45, Jan. 2016.

[23] H. Peng et al., "Performance analysis of IEEE 802.11p DCF for multiplatooning communications with autonomous vehicles," IEEE Trans. Veh. Technol., vol. 66, no. 3, pp. 2485-2498, Mar. 2017.

[24] J. Zhao and G. Cao, "VADD: Vehicle-assisted data delivery in vehicular ad hoc networks," IEEE Trans. Veh. Technol., vol. 57, no. 3, pp. 1910-1922, May 2008.

[25] Z. Khan, P. Fan, and S. Fang, "On the connectivity of vehicular ad hoc network under various mobility scenarios," IEEE Access, vol. 5 , pp. 22559-22565, Oct. 2017.

[26] Y. Zhuang, J. Pan, Y. Luo, and L. Cai, "Time and location-critical emergency message dissemination for vehicular ad-hoc networks," IEEE J. Sel. Areas Commun., vol. 29, no. 1, pp. 187-196, Jan. 2011.

[27] L. Kleinrock, Queueing Systems vol. 1. New York, NY, USA: Wiley, 1975.

[28] K. A. Hafeez, L. Zhao, B. Ma, and J. W. Mark, "Performance analysis and enhancement of the DSRC for VANET's safety applications," IEEE Trans. Veh. Technol., vol. 62, no. 7, pp. 3069-3083, Sep. 2013.

[29] J. Härri, F. Filali, C. Bonnet, and M. Fiore, "VanetMobiSim: Generating realistic mobility patterns for VANETs," in Proc. IEEE VANET, Sep. 2006, pp. 96-97.

[30] C. Sommer, D. Eckhoff, R. German, and F. Dressler, "A computationally inexpensive empirical model of IEEE $802.11 \mathrm{p}$ radio shadowing in urban environments," in Proc. IEEE WONS, Jan. 2011, pp. 84-90.

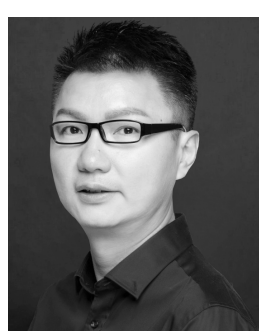

Chen Chen (M'09) received the B.Eng., M.Sc., and Ph.D. degrees in electrical engineering and computer science (EECS) from Xidian University, Xi' an, China, in 2000, 2006, and 2008, respectively. $\mathrm{He}$ is currently an Associate Professor with the Department of EECS, Xidian University. He is also the Director of the engineering technology transfer center with Xi'an in edge computing, and the Associate Director of the Intelligent Transportation Research Laboratory, Xidian University. He was a Visiting Professor of EECS with The University of Tennessee Knoxville, Knoxville, TN, USA, from 2013 to 2014. He has authored/co-authored two books, over 70 scientific papers in international journals and conference proceedings. He has contributed to the development of two copyrighted software systems and invented 40 patents. He is a Senior Member of the China Computer Federation and a member of the ACM and the Chinese Institute of Electronics. He serves as the general chair, the pc chair, and the workshop chair or a TPC member of a number of conferences. 


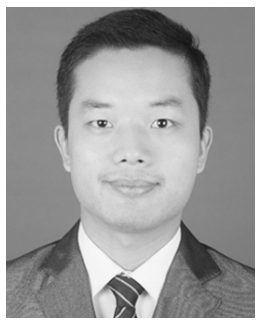

Lei Liu received the B.Eng. degree in communication engineering from Zhengzhou University, Zhengzhou, China, in 2010 and the M.Sc. degree in communication engineering from Xidian University, Xi' an, China, in 2013, where he is currently pursuing the Ph.D. degree. His research interests include intelligent transportation and Internet of Thing.

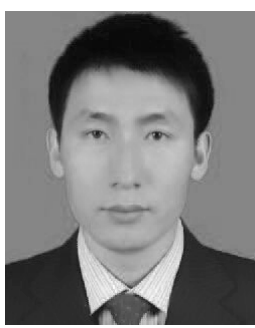

Tie Qiu (M'12-SM'16) received the Ph.D. degree in computer science from Dalian University of Technology in 2012. He is currently a Full Professor with the School of Computer Science and Technology, Tianjin University, China. He was with the School of Software, Dalian University of Technology, as an Assistant Professor in 2008 and an Associate Professor in 2013. He was a Visiting Professor of electrical and computer engineering with Iowa State University, Ames, IA, USA, from 2014 to 2015. He has authored/co-authored nine books, over 100 scientific papers in international journals and conference proceedings, such as IEEE/ACM ToN, IEEE TMC, TII, TIP, TCY, and TITS, IEEE COMMUNICATIONS SURVEYS AND TUTORIALS, IEEE COMMUNICATIONS, IEEE Systems Journal, IEEE IoT Journal, Computer Networks, and the IEEE SMC, ICC, and CSCWD. There are five papers listed as ESI highly cited papers. He has contributed to the development of three copyrighted software systems and invented 10 patents. He is a Senior Member of the China Computer Federation and a Senior Member of the ACM. He serves as the general chair, the program chair, the workshop chair, the publicity chair, the publication chair or a TPC member of a number of international conferences. He serves as an Area Editor for Ad Hoc Networks (Elsevier), an Associate Editor for IEEE ACCESS, Computers and Electrical Engineering (Elsevier), Human-centric Computing and Information Sciences (Springer), and International Journal on AdHoc Networking Systems, and a Guest Editor for Future Generation Computer Systems.

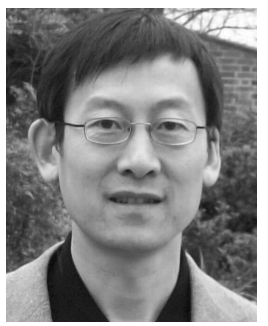

Kun Yang (M'02-SM'12) received the Ph.D. degree from the Department of Electronic and Electrical Engineering, University College London, U.K. He is currently a Full Professor with the School of Computer Science and Electronic Engineering, University of Essex, U.K., and the Head of the Network Convergence Laboratory, Essex. His main research interests include wireless networks/communications, fixed mobile convergence, future Internet technology and network virtualization. He has published over 150 papers in the above research areas. He serves on the editorial boards of both IEEE and non-IEEE journals. He is a fellow of the IET.

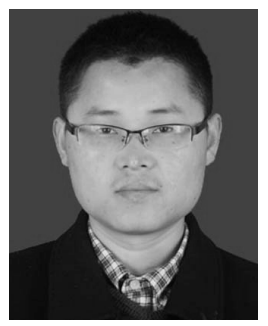

Fengkui Gong (M'12) was born in Shandong, China, in 1979. He received the M.S. and Ph.D. degrees with the Department of Communication Engineering, Xidian University, in 2004 and 2007, respectively. Since 2008 , he has been a Professor with the State Key Laboratory of ISN. His research interests include communication signal processing and digital video broadcasting.

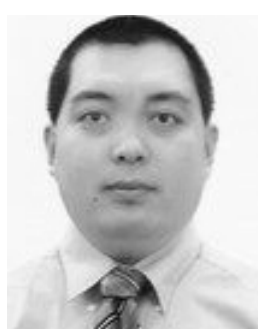

Houbing Song (M'12-SM'14) received the Ph.D. degree in electrical engineering from University of Virginia, Charlottesville, VA, USA, in 2012. In 2017, he joined the Department of Electrical, Computer, Software, and Systems Engineering, Embry-Riddle Aeronautical University, Daytona Beach, FL, USA, where he is currently an Assistant Professor and the Director of the Security and Optimization for Networked Globe Laboratory. He served on the faculty of West Virginia University from 2012 to 2017. In 2007, he was an Engineering Research Associate with the Texas A\&M Transportation Institute. He serves as an Associate Technical Editor for IEEE Communications Magazine. He is an Editor of four books, including Smart Cities: Foundations, Principles and Applications (Hoboken, NJ: Wiley, 2017), Security and Privacy in Cyber-Physical Systems: Foundations, Principles and Applications (Chichester, UK: Wiley-IEEE Press, 2017), Cyber-Physical Systems: Foundations, Principles and Applications (Boston, MA: Academic Press, 2016), and Industrial Internet of Things: Cybermanufacturing Systems (Cham, Switzerland: Springer, 2016). He is an author of over 100 articles. His research interests include cyber-physical systems, cybersecurity and privacy, Internet of Things, edge computing, big data analytics, unmanned aircraft systems, connected vehicle, smart and connected health, and wireless communications and networking.

Dr. Song is a Senior Member of the ACM. He was the very first recipient of the Golden Bear Scholar Award, the highest campus-wide recognition for research excellence at West Virginia University Institute of Technology, in 2016. He was a recipient of the Air Force Research Laboratory's Information Directorate 2018 Visiting Faculty Research Program Award. 